\begin{tabular}{|c|l|}
\hline Title & On a dynamic boundary condition for singular degenerate parabolic equations in a half space \\
\hline Author(s) & Giga, Yoshikazu; Hamamuki, Nao \\
\hline Citation & Hokkaido University Preprint Series in Mathematics, 1110, 1-38 \\
\hline Issue Date & 2018-04_28 \\
\hline DOI & 10.14943/84298 \\
\hline Doc URL & http://hdl.handle.net/2115/70072 \\
\hline Type & bulletin (article) \\
\hline File Information & document_20180428.pdf \\
\hline
\end{tabular}

Instructions for use 


\title{
On a dynamic boundary condition for singular degenerate parabolic equations in a half space
}

\author{
Yoshikazu Giga * and Nao Hamamuki ${ }^{\dagger}$
}

April 28, 2018

\begin{abstract}
We consider the initial value problem for a fully-nonlinear degenerate parabolic equation with a dynamic boundary condition in a half space. Our setting includes geometric equations with singularity such as the level-set mean curvature flow equation. We establish a comparison principle for a viscosity sub- and supersolution. We also prove existence of solutions and Lipschitz regularity of the unique solution. Moreover, relation to other types of boundary conditions is investigated by studying the asymptotic behavior of the solution with respect to a coefficient of the dynamic boundary condition.
\end{abstract}

Key words: dynamic boundary condition; geometric equations; comparison principle; viscosity solutions

Mathematics Subject Classification 2010: 35K20; 35B51; 35D40

\section{Introduction}

Equation We consider the initial value problem for a fully-nonlinear parabolic equation with a dynamic boundary condition of the form

$$
(\mathrm{DB}) \begin{cases}u_{t}(x, t)+F\left(\nabla u(x, t), \nabla^{2} u(x, t)\right)=0 & \text { in } \Omega \times(0, T), \\ u_{t}(x, t)-\beta u_{x_{n}}(x, t)=0 & \text { on } \partial \Omega \times(0, T), \\ u(x, 0)=u_{0}(x) & \text { in } \bar{\Omega} .\end{cases}
$$

Here $u: \bar{\Omega} \times[0, T) \rightarrow \mathbf{R}$ is unknown and $u_{t}=\partial_{t} u, \nabla u=\left(\partial_{x_{i}} u\right)_{i=1}^{n}$ and $\nabla^{2} u=\left(\partial_{x_{i} x_{j}} u\right)_{i, j=1}^{n}$. Moreover, throughout this paper we assume that

- $\Omega:=\left\{x=\left(x^{\prime}, x_{n}\right) \in \mathbf{R}^{n-1} \times \mathbf{R} \mid x_{n}>0\right\}$ is the half space of $\mathbf{R}^{n}$;

\footnotetext{
${ }^{*}$ Graduate School of Mathematical Sciences, The University of Tokyo, 3-8-1 Komaba, Meguro, Tokyo, 153-8914 Japan. E-mail: labgiga@ms.u-tokyo.ac.jp

${ }^{\dagger}$ Department of Mathematics, Hokkaido University, Kita 10, Nishi 8, Kita-Ku, Sapporo, Hokkaido, 0600810, Japan. E-mail: hnao@math.sci.hokudai.ac.jp
} 
- $\beta>0$.

The prime' and the index $n$ are used to represent the first $(n-1)$ components and the last component of a vector in $\mathbf{R}^{n}$, respectively.

Our goal in this paper is to establish a comparison principle, existence and regularity results of viscosity solutions to (DB) when $F=F(p, X)$ is allowed to be singular at $p=0$. Examples include the level-set mean curvature flow operator stated below. We also study the asymptotic behavior of the unique solution as $\beta \rightarrow 0$ and $\beta \rightarrow \infty$.

The case $\beta=0$ appears to be the Dirichlet problem but it is not exactly the same as the traditional Dirichlet problem $u=u_{0}$ on the boundary $\partial \Omega$; see Section 6.3. We also note that the case $\beta<0$ is ill-posed if $n \geqq 2$ as pointed out by Vázquez and Vitillaro [48]. This is why we restrict ourselves for $\beta>0$ although we occasionally mention the case $\beta=0$.

Interface evolution equations Our problem (DB) applies to level-set equations for interface problems, which were first rigorously analyzed by [8] and [17]; the method was introduced by [42] as a numerical scheme and by [43] for physical explanation of scaling laws. We briefly explain the method below. See [29, Chapter 1] for a more detailed description of this approach.

Let us consider evolving (smooth) interfaces $\left\{\Gamma_{t}\right\}_{t \in[0, T)}$ in $\bar{\Omega}$ which divide $\bar{\Omega}$ into two regions $\Omega_{t}^{+}$and $\Omega_{t}^{-}$. See Figure 1 . Denote by $\mathbf{n}=\mathbf{n}(x, t)$ the unit normal vector at $x \in \Gamma_{t}$ from $\Omega_{t}^{+}$to $\Omega_{t}^{-}$. We assume that $\Gamma_{t}$ evolves according to a surface evolution equation

$$
V=f(\mathbf{n}, \nabla \mathbf{n}) \quad \text { on } \Gamma_{t} \cap \Omega \text {. }
$$

Here $V=V(x, t)$ is the normal velocity of $\Gamma_{t}$ at $x$ in the direction of $\mathbf{n}, f$ is a given function, and $-\nabla \mathbf{n}$ is the second fundamental form in the direction of $\mathbf{n}$. To track a motion of $\Gamma_{t}$ we represent it as the zero level-set of an auxiliary function $u(x, t)$. Namely, $\Gamma_{t}=\{u(\cdot, t)=0\}$ and $\Omega_{t}^{ \pm}=\{ \pm u(\cdot, t)>0\}$. Then

$$
V=\frac{u_{t}}{|\nabla u|}, \quad \mathbf{n}=-\frac{\nabla u}{|\nabla u|}, \quad \nabla \mathbf{n}=-\frac{1}{|\nabla u|} Q_{\nabla u}\left(\nabla^{2} u\right),
$$

where

$$
Q_{p}(X)=R_{p} X R_{p} \quad \text { with } R_{p}=I-\frac{p \otimes p}{|p|^{2}}
$$

provided that $u$ is smooth and $\nabla u \neq 0$. Here we write $q \otimes q=\left(q_{i} q_{j}\right)_{i j}$ for $q=\left(q_{i}\right)_{i} \in \mathbf{R}^{n}$, and $|\cdot|$ stands for the standard Euclidean norm in $\mathbf{R}^{n}$. Substituting the above formulas for (1.4), we are led to the parabolic equation (1.1) for $u$ with a (possibly) singular operator $F$ given by

$$
F(p, X)=F_{f}(p, X)=-|p| f\left(-\frac{p}{|p|},-\frac{1}{|p|} Q_{p}(X)\right) .
$$

The resulting equation (1.1) is often called a level-set equation. The operator $F$ derived in this way possesses a kind of scaling property called geometricity; see (F5) in Section 4.3.

A typical example of such surface evolution equations in our mind is the mean curvature flow equation given by

$$
V=H \quad \text { on } \Gamma_{t} \cap \Omega
$$


with the mean curvature $H=-\left(\operatorname{div}_{\Gamma_{t}} \mathbf{n}\right)(x)$ of $\Gamma_{t}$ at $x$. In this case the associated equation $(1.1)$ is

$$
u_{t}(x, t)-|\nabla u(x, t)| \operatorname{div}\left(\frac{\nabla u(x, t)}{|\nabla u(x, t)|}\right)=0 \quad \text { in } \Omega \times(0, T)
$$

with $F$ defined as

$$
F(p, X)=-\operatorname{tr}\left\{\left(I-\frac{p \otimes p}{|p|^{2}}\right) X\right\} .
$$

In the level-set formulation, our dynamic boundary condition (1.2) corresponds to the following boundary condition for evolving surfaces:

$$
v_{b}=\beta \cot \theta \quad \text { on } b \Gamma_{t}:=\Gamma_{t} \cap \partial \Omega .
$$

Here $v_{b}=v_{b}(x, t)$ is the normal velocity of $b \Gamma_{t}$ on the boundary $\partial \Omega$ at $x \in b \Gamma_{t}$, and $\theta$ is the contact angle at $x \in b \Gamma_{t}$ formed by $\partial \Omega$ and $\Gamma_{t}$, or equivalently the angle between two vectors $e_{n} \in(0, \ldots 0,1) \in \mathbf{R}^{n}$ and $-\mathbf{n}$ at $x \in b \Gamma_{t}$. Since they are given as

$$
v_{b}=\frac{V}{\sin \theta}=\frac{u_{t}}{|\nabla u| \sin \theta}, \quad \cos \theta=\left\langle e_{n},-\mathbf{n}\right\rangle=\frac{u_{x_{n}}}{|\nabla u|},
$$

where $\langle\cdot, \cdot\rangle$ is the standard Euclidean inner product in $\mathbf{R}^{n}$, we see that (1.10) yields (1.2).

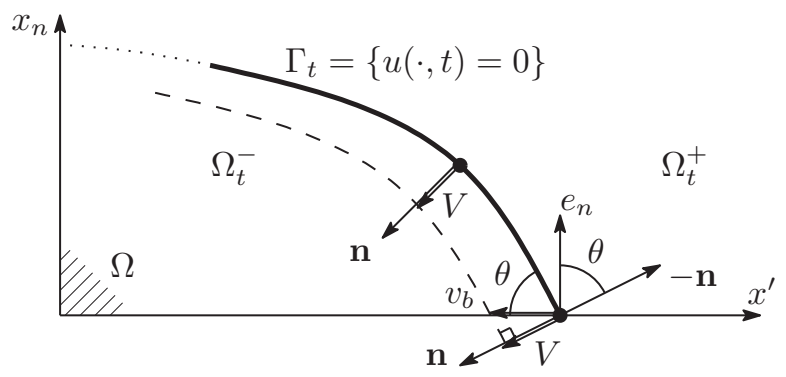

Figure 1: Interface problem

For (1.8) and more general singular geometric equations, a unique existence result for viscosity solutions is obtained by [8] and [17] in the case where there is no boundary, that is, $\Omega=\mathbf{R}^{n}$. When $\Omega$ is a domain, a unique existence of viscosity solutions for singular equations is well studied for Neumann type boundary conditions; see [44, 45, 6, 38]. See also [47, 36] for Dirichlet boundary problems, but the domain $\Omega$ is assumed to be strict mean-convex which excludes the half space. However, such a well-posedness result for singular equations like (1.8) has not been known for a dynamic boundary condition even if the boundary is flat like our case. This paper provides a first attempt in this problem.

Our typical problem (1.8) with (1.2) is obtained, at least formally, as a sharp interface limit of the Allen-Cahn equation

$$
v_{t}^{\varepsilon}(x, t)-\Delta v^{\varepsilon}(x, t)+\frac{1}{\varepsilon^{2}} W^{\prime}\left(v^{\varepsilon}(x, t)\right)=0 \quad \text { in } \Omega \times(0, T)
$$


with the same dynamic boundary condition

$$
v_{t}^{\varepsilon}(x, t)-\beta v_{x_{n}}^{\varepsilon}(x, t)=0 \quad \text { on } \partial \Omega \times(0, T) .
$$

Here $W(r)=\left(1-r^{2}\right)^{2} / 4$ is a double-well potential. See, e.g., [26, 9] and references therein for the Allen-Cahn equation with a dynamic boundary condition. Also, the authors of [31] study Brakke flows with Dirichlet or dynamic boundary condition obtained by the limit of the Allen-Cahn equation.

To see the derivation of (1.8) and (1.2), let us suppose as usual that the solution $v^{\varepsilon}$ is of the form

$$
v^{\varepsilon}(x, t)=q\left(\frac{d(x, t)}{\varepsilon}\right)
$$

where $q$ is the standing wave solution to $q^{\prime \prime}(r)-W^{\prime}(q(r))=0$ in $\mathbf{R}$ with $q( \pm \infty)= \pm 1$ and $d$ is a smooth function such that $\{d=0\}=\left\{v^{\varepsilon}=0\right\}$. On one hand, the equation (1.8) for $d$ is derived in a usual way by substituting the derivatives of $v^{\varepsilon}$ for (1.11). Indeed, we then have

$$
\frac{1}{\varepsilon} q^{\prime}\left(\frac{d}{\varepsilon}\right)\left(d_{t}-\Delta d\right)-\frac{1}{\varepsilon^{2}} q^{\prime \prime}\left(\frac{d}{\varepsilon}\right)\left(|\nabla d|^{2}-1\right)=0 .
$$

This implies that $d_{t}-\Delta d=0$ and $|\nabla d|^{2}=1$, and hence

$$
\begin{aligned}
d_{t}-\operatorname{tr}\left\{\left(I-\frac{\nabla d \otimes \nabla d}{|\nabla d|^{2}}\right) \nabla^{2} d\right\} & =d_{t}-\Delta d+\sum_{i, j=1}^{n} d_{x_{i}} d_{x_{j}} d_{x_{i} x_{j}} \\
& =0+\sum_{i=1}^{n} d_{x_{i}} \partial_{x_{i}}\left(|\nabla d|^{2}\right)=0 .
\end{aligned}
$$

Since $|\nabla d|=1$, we see that $d$ is a distance function to $\{d(\cdot, t)=0\}=\left\{v^{\varepsilon}(\cdot, t)=0\right\}$. On the other hand, it follows from (1.12) that

$$
\frac{1}{\varepsilon} q^{\prime}\left(\frac{d}{\varepsilon}\right)\left(d_{t}-\beta d_{x_{n}}\right)=0,
$$

which gives (1.2) for $d$. In a strict sense, it is known that the distance function is a viscosity supersolution of the heat equation. This is shown in [16] and [39] for the case with no boundary and the case of the Neumann boundary condition, respectively. By using this fact, $[16,39]$ prove that the zero level-set of a solution to the Allen-Cahn equation converges to a mean curvature flow. Unfortunately, it seems that a similar technique does not work for (DB). In fact, we show in Example 2.5 that the distance function may not be a supersolution of the heat equation in $\Omega$.

A similar type of boundary conditions to (1.2) can be found in [4]; it is of the form

$$
v_{b}=f \cos \theta+g
$$

with $f$ free energy of the interface and $g$ the difference between the free energies of two parts of $\partial \Omega$ separated by the interface. The derivation of (1.13) is based on a capillary force balance, dissipation inequality etc. 
Literature overview We review a few typical known results on dynamic boundary value problems related to our problem. This list is not exhaustive at all.

For fully nonlinear parabolic equations without a singularity at $\nabla u=0$, Barles established a quite general comparison result in [5, Section II] and [6, Section 3] for a general nonlinear dynamic boundary condition; see also [7] for the large time behavior of the solution. However, as is stated in [6, Comment after Theorem 3.2], a similar choice of a test function to the non-singular case does not work when we prove the comparison principle for singular equations. A difficulty is a control of $\left|\nabla_{x} \phi-\nabla_{y} \phi\right|$ for a test function $\phi=\phi(x, t, y, s)$ in the procedure of doubling variables. In this paper, we carry out a different approach based on a perturbation of a test function employed in $[33,27,28]$ rather than modification of test functions given in $[5,6]$.

In the context of viscosity solutions, the papers $[12,2]$ study dynamic boundary problems for first order equations. Motivated by a mean field models of superconductivity and a surface evolution problem, the authors of [12] study a Hamilton-Jacobi equation under a dynamic boundary condition without the spatial derivative of the unknown function in one space dimensional case. A comparison principle and an existence theorem are established. The paper [2] is concerned with the asymptotic behavior of solutions to a dynamic boundary problem for the eikonal equation

$$
\varepsilon u_{t}(x, t)+|\nabla u(x, t)|=1 \text { in } D \times(0, \infty)
$$

with a bounded domain $D$. The limit of the solution $u$ as $\varepsilon \rightarrow+0$ is investigated. Recent work $[24,35]$ also study such a vanishing time derivative of the equation for second order equations. In $[34,35]$ a deterministic game interpretation is proposed for dynamic boundary value problems of the mean curvature flow equation (1.8) and fully nonlinear parabolic equations.

The paper [1] proves a short-time existence and uniqueness of solutions to equations for the volume-preserving mean curvature flow and Willmore flow. See also $[14,11,48,46]$ for various other studies of a dynamic boundary problem for parabolic equations; the heat equation [48], semilinear equations [46], quasilinear equations [14] and higher order equations [11]. These work emphasize construction of unique regular solutions.

The theory for an elliptic equation of the form

$$
\begin{cases}-\Delta u(x, t)=f(u(x, t)) & \text { in } D \times(0, T), \\ u_{t}(x, t)+\langle\nu, \nabla u(x, t)\rangle=g(u(x, t)) & \text { on } \partial D \times(0, T),\end{cases}
$$

where $\nu$ is a unit outward normal vector to $\partial D$, has also been developed. When $D$ is the half space $\Omega$, the paper [3] establishes a blow-up theorem of Fujita type when $f \equiv 0$ and $g(u)=u^{p}$. See also $[40,41,18]$ for extension of the result and the asymptotic behavior of solutions. When $f(u)=u^{p}$ (or more general functions) and $g \equiv 0$, Fila, Ishige and Kawakami investigated (non-)existence of solutions, the large time behavior, minimal solutions and so on; see $[19,20,21,22]$. We also refer the reader to $[13,15,25]$ for problems in a general bounded domain $D$ and [23] for problems in an exterior domain $D$.

Results Our first result of this paper is a comparison principle presented in Section 3. It applies to a viscosity subsolution $u$ and a viscosity supersolution $v$ of (DB) with a singular $F$ 
which is not necessarily geometric. The theorem is proved under a suitable decay condition for $u-v$ at infinity, which is satisfied if, for example, $u$ and $v$ are constant at infinity. Namely, both $u(\cdot, t)-\alpha$ and $v(\cdot, t)-\alpha$ have a compact support for some $\alpha \in \mathbf{R}$. This assumption is usual and not restrictive in the level-set approach when we study a motion of bounded interfaces $([8,17])$. As a corollary of the comparison result, we obtain uniqueness of solutions when the initial data is constant at infinity.

Our idea to handle a singularity together with a dynamic boundary condition is as follows: As usual we study a maximum point $\hat{Z}$ of

$$
\Phi(x, t, y, s)=u(x, t)-v(y, s)-\phi(x, t, y, s)
$$

with a smooth test function $\phi$. A standard choice of $\phi$ involves $|x-y|^{4}$ to guarantee that $\nabla^{2} \phi=O$ whenever $\nabla \phi=0$; see the assumption (F3). Now, we add a perturbation parameter $\zeta \in \mathbf{R}$ to $x_{n}$-variable, which is the normal direction to the boundary of $\Omega$, and put $\mid x^{\prime}-$ $\left.y^{\prime}\right|^{4}+\left|x_{n}-y_{n}-\zeta\right|^{2}$ into $\phi=\phi^{\zeta}$. Such a perturbation of a test function is first employed in [33, Theorem 2.2]; see also [27, Comparison Principle (Section 1.2), Proposition 7.6] and [28, Theorem 4.1]. We further add a suitable term so that $\phi$ violates the boundary condition (1.2).

The classical argument is applicable if there is some $\zeta$ such that $\nabla \phi \neq 0$ at the maximum point $\hat{Z}=\hat{Z}^{\zeta}$ since the singularity does not affect. A difficulty arises if it is not the case. Then it turns out that, we are able to reduce the number of the variables of the test function by using the relation $\phi_{x_{n}}=0$. In this way, we are led to define a reduced test function $\rho$ which no longer depends on $x_{n}, y_{n}$ and $\zeta$. It also turns out that a maximum point of the associated function $\Theta(x, t, y, s)=u(x, t)-v(y, s)-\rho(x, t, y, s)$ is unchanged from $\hat{Z}^{0}$. This fact is guaranteed by using the constancy lemma (Lemma 2.3). Accordingly, we have $\nabla \rho=0$ and $\nabla^{2} \rho=O$ at $\hat{Z}^{0}$, which concludes the theorem. This approach is sometimes called a flattening argument $[28,32]$. Unfortunately, it seems to be non-trivial to extend this approach for more general boundary conditions rather than the linear condition (1.2) since the derivatives of $\phi$ are not well controlled.

Existence results of viscosity solutions are proven in Section 4 by employing Perron's method for viscosity solutions ([37]). A crucial step is to construct barrier functions which are a viscosity sub- and supersolution satisfying the initial data. We first construct such barriers for a general uniformly continuous initial data, which gives a viscosity solution of (DB). We further construct refined barriers for an initial data being constant at infinity so that the barriers are also constant at infinity. A unique existence result of viscosity solutions of (DB) is thus established for such an initial data. Our unique existence result yields unique global-in-time existence of a generalized solution (level-set flow) of interface evolution equations (1.4) with (1.10).

We also study Lipschitz regularity of the unique solution $u$ in Section 5. In the proof of Theorem 5.3, on one hand, we apply the comparison principle to $u$ with its shift both in space and time variables to get Lipschitz estimates with respect to $x^{\prime}$ and $t$. On the other hand, for the estimate with respect to $x_{n}$-variable, we employ the method of doubling variables like the proof of the comparison principle. We again study a function of the form (1.14) with $u$ instead of $v$. Such a method has been used in the literature; see, e.g., [5, Theorem II.3]. These Lipschitz estimates are derived for initial data $u_{0}$ in $C^{1+1}$-class, which 
is the same regularity assumption as in [5, Theorem II.3]. We further discuss in Section 5.2 a sufficient condition which guarantees that the Lipschitz constant is independent of $\beta$.

In Section 6 relation to other types of boundary conditions is investigated. We study the asymptotic behavior of the unique solution $u=u^{\beta}$ of (DB) as $\beta \rightarrow \infty$ and $\beta \rightarrow 0$. At least formally, these limits are expected to solve

$$
-u_{x_{n}}(x, t)=0 \quad \text { on } \partial \Omega \times(0, T) \quad \text { and } \quad u_{t}(x, t)=0 \quad \text { on } \partial \Omega \times(0, T),
$$

respectively. In other words, new boundary conditions are of the Neumann type and the "Dirichlet" type. This formal observation can be justified by stability results for the halfrelaxed limits of viscosity solutions, provided that the comparison principle for the limit problem holds. For the Neumann case, the convergence is established in Section 6.1 since the comparison principle is a classical result $([45])$, while it fails in general for the Dirichlet type. For this reason, in Section 6.2 and Section 6.3 we give some sufficient conditions that imply the convergence of $u^{\beta}$ to the solution of the limit problem of the Dirichlet type.

By a change of variables, it is possible to treat a more general boundary condition of the form

$$
u_{t}(x, t)-\left\langle\gamma, \nabla_{x^{\prime}} u(x, t)\right\rangle-\beta u_{x_{n}}(x, t)=0 \quad \text { on } \partial \Omega \times(0, T)
$$

with a vector $\gamma \in \mathbf{R}^{n-1}$. In fact, $u(x, t)$ is a viscosity solution of (1.1), (1.15) and (1.3) if and only if $v(x, t):=u\left(x^{\prime}-\gamma t, x_{n}, t\right)$ is a viscosity solution of (DB). For this reason, we only study the simpler problem (DB) and give results for (DB) in this paper.

This paper is organized as follows: Section 2 is devoted to preparation. We give a definition of viscosity solutions, a key lemma and some examples. In Section 3 we establish a comparison principle for a viscosity sub- and supersolution of (DB). This implies a uniqueness of solutions while existence of solutions is discussed in Section 4. As an application, we give a typical result for interface evolution equations with the boundary condition (1.10). Section 5 is concerned with a Lipschitz regularity of the unique solution. Finally, in Section 6 we study the asymptotic behavior of the solution to (DB) as $\beta \rightarrow 0$ and $\beta \rightarrow \infty$. We prove a convergence to a solution of the limit problem.

\section{Preliminaries}

\subsection{Definition of viscosity solutions}

Let $K \subset \mathbf{R}^{N}$ be a subset. For a function $h: K \rightarrow \mathbf{R}$, its upper semicontinuous envelope $h^{*}$ and lower semicontinuous envelope $h_{*}$ are defined as

$$
h^{*}(x):=\limsup _{K \ni y \rightarrow x} h(y), \quad h_{*}(x):=\liminf _{K \ni y \rightarrow x} h(y) \quad(x \in \bar{K}) .
$$

We list assumptions on the function $F:\left(\mathbf{R}^{n} \backslash\{0\}\right) \times \mathbf{S}^{n} \rightarrow \mathbf{R}$ appearing in (1.1). Here $\mathbf{S}^{n}$ stands for the space of real $n \times n$ symmetric matrices with the usual ordering. Namely, we write $X \leqq Y$ if $\langle(Y-X) \xi, \xi\rangle \geqq 0$ for all $\xi \in \mathbf{R}^{n}$.

(F1) $F$ is continuous in $\left(\mathbf{R}^{n} \backslash\{0\}\right) \times \mathbf{S}^{n}$. 
(F2) $F$ is degenerate elliptic, i.e, $F(p, X) \geqq F(p, Y)$ for all $p \in \mathbf{R}^{n} \backslash\{0\}$ and $X, Y \in \mathbf{S}^{n}$ such that $X \leqq Y$.

(F3) $-\infty<F_{*}(0, O)=F^{*}(0, O)<\infty$.

Throughout this paper we assume (F1)-(F3). We remark that it follows from (F2) that $F^{*}(0, X) \geqq F^{*}(0, Y)$ and $F_{*}(0, X) \geqq F_{*}(0, Y)$ if $X \leqq Y$.

To give a definition of viscosity solutions, we introduce a notion of parabolic semi-jets $\mathcal{P}^{2, \pm} u\left(x_{0}, t_{0}\right)$. Let $\left(x_{0}, t_{0}\right) \in \bar{\Omega} \times(0, T)$. We define $\mathcal{P}^{2,+} u\left(x_{0}, t_{0}\right)$ (resp. $\left.\mathcal{P}^{2,-} u\left(x_{0}, t_{0}\right)\right)$ as the set of $\left(\left(\nabla \phi\left(x_{0}, t_{0}\right), \phi_{t}\left(x_{0}, t_{0}\right)\right), \nabla^{2} \phi\left(x_{0}, t_{0}\right)\right) \in \mathbf{R}^{n} \times \mathbf{R} \times \mathbf{S}^{n}$ with $\phi \in C^{2,1}(\bar{\Omega} \times(0, T)), C^{2}$-class in $x$ and $C^{1}$-class in $t$, such that $u-\phi$ attains a local maximum (resp. local minimum) at $\left(x_{0}, t_{0}\right)$ over $\bar{\Omega} \times(0, T)$. By definition we have $\mathcal{P}^{2,-} u\left(x_{0}, t_{0}\right)=-\mathcal{P}^{2,+}(-u)\left(x_{0}, t_{0}\right)$. For equivalent definitions and some basic properties, see [29, Chapter 3.2.1] for instance.

Definition 2.1 (Viscosity solution). Let $u: \Omega \times[0, T) \rightarrow \mathbf{R}$. We say that $u$ is a viscosity subsolution (resp. a viscosity supersolution) of (1.1) and (1.2) if $u^{*}<\infty$ (resp. $u_{*}>-\infty$ ) in $\bar{\Omega} \times(0, T)$ and if for all $\left(x_{0}, t_{0}\right) \in \bar{\Omega} \times(0, T)$ and $((p, \tau), X) \in \mathcal{P}^{2,+} u^{*}\left(x_{0}, t_{0}\right)$ (resp. $\left.((p, \tau), X) \in \mathcal{P}^{2,-} u^{*}\left(x_{0}, t_{0}\right)\right)$, we have

$$
\begin{cases}\tau+F_{*}(p, X) \leqq 0 \quad\left(\text { resp. } \tau+F^{*}(p, X) \geqq 0\right) & \text { if } x_{n}>0 \\ \tau+F_{*}(p, X) \leqq 0 \text { or } \tau-\beta p_{n} \leqq 0 & \text { if } x_{n}=0 . \\ \text { (resp. } \left.\tau+F^{*}(p, X) \geqq 0 \text { or } \tau-\beta p_{n} \geqq 0\right) & \end{cases}
$$

If $u$ further satisfies $u^{*}(\cdot, 0) \leqq u_{0}$ (resp. $\left.u_{*}(\cdot, 0) \geqq u_{0}\right)$ in $\bar{\Omega}$, then we say that $u$ is a viscosity subsolution (resp. a viscosity supersolution) of (DB). A viscosity solution is a function which is both a viscosity subsolution and a viscosity supersolution.

Remark 2.2. Assume that $u$ is a viscosity subsolution of (1.1) and (1.2) and let $\left(x_{0}, t_{0}\right) \in$ $\partial \Omega \times(0, T)$. If $((p, \tau), X) \in \mathcal{P}^{2,+} u^{*}\left(x_{0}, t_{0}\right)$ and if the boundary condition (1.2) is violated, namely $\tau-\beta p_{n}>0$, then the inequality $\tau+F_{*}(p, X) \leqq 0$ should be fulfilled. This is clear by the definition of subsolutions.

The same thing is true for an element of the extended parabolic semi-jet $\overline{\mathcal{P}}^{2,+} u^{*}\left(x_{0}, t_{0}\right)$, which is defined as the set of $((p, \tau), X) \in \mathbf{R}^{n} \times \mathbf{R} \times \mathbf{S}^{n}$ such that

there exist $\left\{\left(x_{m}, t_{m}\right)\right\}_{m=1}^{\infty} \subset \bar{\Omega} \times(0, T)$ and $\left\{\left(\left(p_{m}, \tau_{m}\right), X_{m}\right)\right\}_{m=1}^{\infty} \subset \mathbf{R}^{n} \times \mathbf{R} \times \mathbf{S}^{n}$ such that $\left(\left(p_{m}, \tau_{m}\right), X_{m}\right) \in \mathcal{P}^{2,+} u^{*}\left(x_{m}, t_{m}\right)$ and, as $m \rightarrow \infty,\left(x_{m}, t_{m}\right) \rightarrow\left(x_{0}, t_{0}\right)$, $\left(\left(p_{m}, \tau_{m}\right), X_{m}\right) \rightarrow((p, \tau), X)$ and $u^{*}\left(x_{m}, t_{m}\right) \rightarrow u^{*}\left(x_{0}, t_{0}\right)$.

Indeed, if $((p, \tau), X) \in \overline{\mathcal{P}}^{2,+} u^{*}\left(x_{0}, t_{0}\right)$ and $\tau-\beta p_{n}>0$, then $\tau_{m}-\beta\left(p_{m}\right)_{n}>0$ for sufficiently large $m$. This implies that $\tau_{m}+F_{*}\left(p_{m}, X_{m}\right) \leqq 0$, and by sending $m \rightarrow \infty$ we obtain $\tau+F_{*}(p, X) \leqq 0$. Clearly, a similar assertion holds for a viscosity supersolution $u$ and the extended parabolic semi-jet $\overline{\mathcal{P}}^{2,-} u_{*}\left(x_{0}, t_{0}\right)=-\overline{\mathcal{P}}^{2,+}\left(-u_{*}\right)\left(x_{0}, t_{0}\right)$. 


\subsection{Constancy lemma}

We state a generalized version of the constancy lemma appearing in [33, Section 4, Case $2 \mathrm{~b}]$ and [27, Lemma 7.5]. The lemma plays an important role in the proof of a comparison principle when we perturb a test function to avoid singularities of equations. In Lemma 2.3 below, the variables of $\phi$ are expanded so that it depends on $\rho$ while, in [33, 27], $\phi$ is a function of only $r$. The proof is almost the same as the literature, but we give it for the reader's convenience.

Lemma 2.3 (Constancy lemma). Let $K \subset \mathbf{R}^{N}$ be a bounded set and $G \subset \mathbf{R}^{d}$ be a bounded domain with $1 \leqq d<N$. Let $h: K \rightarrow \mathbf{R} \cup\{-\infty\}$ be a function such that $h \not \equiv-\infty$, and let $\phi=\phi(r, \rho): \mathbf{R}^{N} \rightarrow \mathbf{R}$, where $(r, \rho) \in \mathbf{R}^{d} \times \mathbf{R}^{N-d}$, be a $C^{2}$-function with respect to $r$. For each $\zeta \in G$ we define

$$
H^{\zeta}(r, \rho):=h(r, \rho)-\phi(r-\zeta, \rho) \quad \text { for }(r, \rho) \in K .
$$

If for each $\zeta \in G$ there is a maximizer $\left(r^{\zeta}, \rho^{\zeta}\right) \in K$ of $H^{\zeta}$ over $K$ such that $\nabla_{r} \phi\left(r^{\zeta}-\zeta, \rho^{\zeta}\right)=$ 0 , then $\tilde{h}(\zeta):=H^{\zeta}\left(r^{\zeta}, \rho^{\zeta}\right)$ is constant in $G$.

Proof. Fix $\zeta, \eta \in G$ which satisfy $\theta \zeta+(1-\theta) \eta \in G$ for all $\theta \in[0,1]$. By the definitions of functions $\tilde{h}, H^{\eta}, H^{\zeta}$ and the maximality at $\left(r^{\zeta}, \rho^{\zeta}\right)$, we observe

$$
\begin{aligned}
\tilde{h}(\eta) & =H^{\eta}\left(r^{\eta}, \rho^{\eta}\right)=h\left(r^{\eta}, \rho^{\eta}\right)-\phi\left(r^{\eta}-\eta, \rho^{\eta}\right) \\
& =\left\{h\left(r^{\eta}, \rho^{\eta}\right)-\phi\left(r^{\eta}-\zeta, \rho^{\eta}\right)\right\}+\phi\left(r^{\eta}-\zeta, \rho^{\eta}\right)-\phi\left(r^{\eta}-\eta, \rho^{\eta}\right) \\
& =H^{\zeta}\left(r^{\eta}, \rho^{\eta}\right)+\phi\left(r^{\eta}-\zeta, \rho^{\eta}\right)-\phi\left(r^{\eta}-\eta, \rho^{\eta}\right) \\
& \leqq \tilde{h}(\zeta)+\phi\left(r^{\eta}-\zeta, \rho^{\eta}\right)-\phi\left(r^{\eta}-\eta, \rho^{\eta}\right) .
\end{aligned}
$$

We next apply Taylor's theorem to $\phi$ with center $\left(r^{\eta}-\eta, \rho^{\eta}\right)$. Then, by the assumption that $\nabla_{r} \phi\left(r^{\eta}-\eta, \rho^{\eta}\right)=0$, we see that

$$
\phi\left(r^{\eta}-\zeta, \rho^{\eta}\right)=\phi\left(r^{\eta}-\eta, \rho^{\eta}\right)+\frac{1}{2}\left\langle\nabla_{r}^{2} \phi\left(r^{\eta}-\{\theta \zeta+(1-\theta) \eta\}, \rho^{\eta}\right)(\eta-\zeta),(\eta-\zeta)\right\rangle
$$

for some $\theta \in[0,1]$. Now, by the boundedness of $K$ and $G$, there is a compact set $K_{G}$ in $\mathbf{R}^{N}$ which covers $\{(r-\zeta, \rho) \mid(r, \rho) \in K, \zeta \in G\}$. Fix such $K_{G}$ and define $C:=\left(\max _{K_{G}}\left\|\nabla_{r}^{2} \phi\right\|\right) / 2$. Here $\|X\|:=\sup _{p \in \mathbf{R}^{n},|p| \leqq 1}|X p|$ for $X \in \mathbf{S}^{n}$. It then follows that

$$
\phi\left(r^{\eta}-\zeta, \rho^{\eta}\right) \leqq \phi\left(r^{\eta}-\eta, \rho^{\eta}\right)+C|\eta-\zeta|^{2},
$$

and so $\tilde{h}(\eta)-\tilde{h}(\zeta) \leqq C|\eta-\zeta|^{2}$ by (2.1). Changing the role of $\zeta$ and $\eta$ yields

$$
|\tilde{h}(\eta)-\tilde{h}(\zeta)| \leqq C|\eta-\zeta|^{2}
$$

This gives $\nabla \tilde{h}=0$ in $G$. Since $G$ is connected, we conclude that $\tilde{h}$ is constant in $G$. 


\section{$2.3 \quad$ Examples}

Example 2.4. We study (DB) for the mean curvature flow equation (1.8). For simplicity we let $n=2$. Let $C \in \mathbf{R}$ be a constant and define

$$
u\left(x_{1}, x_{2}, t\right)=C-x_{1}^{2}-\left(x_{2}+\frac{1}{\beta}\right)^{2}-2 t .
$$

Then $u$ is a classical solution (and hence a viscosity solution; see Proposition 4.2) of (DB) with $u_{0}(x)=C-x_{1}^{2}-\left(x_{2}+(1 / \beta)\right)^{2}$. In fact, it is known that the zero level-set of such a function $u$ describes a shrinking circle by the mean curvature; see [29, Chapter 1.7.1]. One can check that $u$ solves (1.1) by direct calculations. It is also easy to check that the boundary condition (1.2) is satisfied since we have $u_{t}(x, t)=-2$ and $u_{x_{2}}(x, t)=-2\left(x_{2}+(1 / \beta)\right)$.

Assume now that $C>1 / \beta^{2}$, so that the zero level-set of $u(\cdot, t)$ in $\bar{\Omega}$, denoted by $\Gamma_{t}$, is not empty for a short time. Then $\Gamma_{t}$ is an arc of a circle of radius $r(t)=\sqrt{C-2 t}$ centered at $Q=(0,-1 / \beta)$, and it connects two points $P_{t}^{ \pm}=\left( \pm \sqrt{C-\left(1 / \beta^{2}\right)-2 t}, 0\right)$. See Figure 2 . An extinction time of $\Gamma_{t}$ is $T^{*}=\left(C-\left(1 / \beta^{2}\right)\right) / 2$. Namely, $\Gamma_{t} \neq \emptyset$ if and only if $t \in\left[0, T^{*}\right]$.

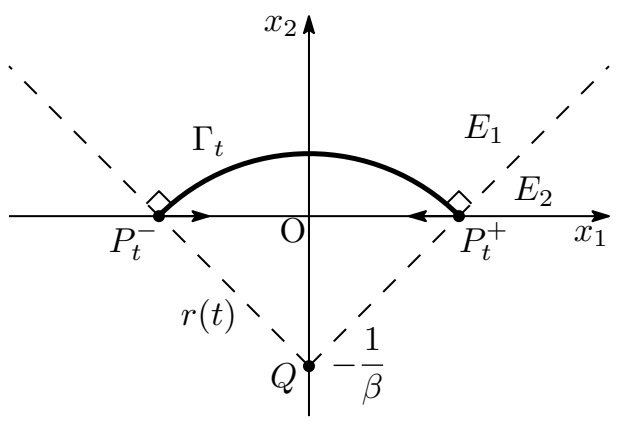

Figure 2: Shrinking arcs

Example 2.5. We revisit Example 2.4 and study the signed distance function $d(x, t)$ to $\Gamma_{t}$, which is given by

$$
d(x, t)= \begin{cases}\operatorname{dist}\left(x, \Gamma_{t}\right) & \text { outside } \Gamma_{t}, \\ -\operatorname{dist}\left(x, \Gamma_{t}\right) & \text { else. }\end{cases}
$$

We show that $d$ is NOT a viscosity supersolution of

$$
d_{t}(x, t)-\Delta d(x, t)=0 \quad \text { in } D^{+},
$$

where $D^{+}=\left\{(x, t) \in \Omega \times\left(0, T^{*}\right) \mid d(x, t)>0\right\}$. This fact shows that our dynamic boundary problem is significantly different from a problem posed in the whole space ([16, Theorem 2.2]) and the Neumann boundary value problem ([39, Lemma 4]).

We use the same notation as in Example 2.4. Let us first calculate the value of $d$ in $\bar{\Omega} \times\left[0, T^{*}\right)$. We divide this set into the following two parts:

$$
\begin{aligned}
& E_{1}:=\left\{(x, t) \in \bar{\Omega} \times\left[0, T^{*}\right) \mid x_{2} \geqq \frac{1}{\beta}\left(\frac{\left|x_{1}\right|}{\left|P_{t}^{ \pm}\right|}-1\right)\right\}, \\
& E_{2}:=\left(\bar{\Omega} \times\left[0, T^{*}\right)\right) \backslash E_{1} .
\end{aligned}
$$


(See Figure 2 again.) Note that int $E_{2} \subset D^{+}$.

If $(x, t) \in E_{1}$, then the distance from $x$ to $\Gamma_{t}$ is achieved at the perpendicular foot on $\Gamma_{t}$, and so the situation is the same as that in [16]. We have

$$
d(x, t)=|x-Q|-r(t),
$$

which implies that

$$
d_{t}(x, t)-\Delta d(x, t)=-r^{\prime}(t)-\frac{1}{|x-Q|}=\frac{1}{r(t)}-\frac{1}{|x-Q|}=\frac{1}{r(t)}-\frac{1}{d(x, t)+r(t)} .
$$

This shows that $d$ is a supersolution of $(2.2)$ in $D^{+} \cap$ int $E_{1}$ in the classical sense.

We next let $(x, t) \in E_{2}$. Then geometric observation shows that the distance from $x$ to $\Gamma_{t}$ is achieved at $P_{t}^{ \pm}$if $\pm x_{1}>0$. Namely,

$$
d(x, t)=\left|x-P_{t}^{ \pm}\right|=\sqrt{\left(x_{1} \mp \sqrt{C-\frac{1}{\beta^{2}}-2 t}\right)^{2}+x_{2}^{2}} \text { if } \pm x_{1}>0 .
$$

By this we find

$$
\begin{aligned}
d_{t}(x, t)-\Delta d(x, t) & =\frac{1}{\left|x-P_{t}^{ \pm}\right|}\left(\frac{\left|x_{1}\right|}{\left|P_{t}^{ \pm}\right|}-1\right)-\frac{1}{\left|x-P_{t}^{ \pm}\right|} \\
& =\frac{1}{\left|x-P_{t}^{ \pm}\right|}\left(\frac{\left|x_{1}\right|}{\left|P_{t}^{ \pm}\right|}-2\right) .
\end{aligned}
$$

This implies that $d_{t}(x, t)-\Delta d(x, t)<0$ for any $(x, t) \in E_{2}$ such that $\left|x_{1}\right|<2\left|P_{t}^{ \pm}\right|$. Therefore $d$ is not a supersolution of (2.2) in the whole of $D^{+}$.

Note that the current $d$ is not a $C^{2}$-function in $x$ on $\left(\Omega \times\left(0, T^{*}\right)\right) \cap \partial E_{1}$ since

$$
\Delta d(x, t)= \begin{cases}\frac{1}{d(x, t)+r(t)} & \text { if }(x, t) \in \operatorname{int} E_{1}, \\ \frac{1}{d(x, t)} & \text { if }(x, t) \in \operatorname{int} E_{2} .\end{cases}
$$

Though $d$ is not a supersolution of $(2.2)$ even on $(\partial \Omega \times(0, T)) \cap E_{2}$, we see that $d$ satisfies the dynamic boundary condition (1.2). Indeed, for any $(x, t) \in E_{2}$, we have

$$
d_{t}(x, t)=\frac{1}{\left|x-P_{t}^{ \pm}\right|}\left(\frac{\left|x_{1}\right|}{\left|P_{t}^{ \pm}\right|}-1\right)>0, \quad d_{x_{2}}(x, t)=\frac{x_{2}}{\left|x-P_{t}^{ \pm}\right|},
$$

which gives $d_{t}(x, t)-\beta d_{x_{2}}(x, t)>0$ if $x_{2}=0$.

\section{Comparison principle}

We denote by $B_{R}(0)$ the open ball in $\mathbf{R}^{n}$ of radius $R>0$ with center at the origin. Our comparison result is stated as follows: 
Theorem 3.1 (Comparison principle). Let $u: \bar{\Omega} \times[0, T) \rightarrow \mathbf{R}$ be a viscosity subsolution of (DB) which is assumed to be bounded from above. Let $v: \bar{\Omega} \times[0, T) \rightarrow \mathbf{R}$ be a viscosity supersolution of (DB) which is assumed to be bounded from below. Assume that

$$
\lim _{R \rightarrow \infty} \sup \left\{u^{*}(x, t)-v_{*}(x, t) \mid(x, t) \in\left(\bar{\Omega} \backslash B_{R}(0)\right) \times[0, T)\right\} \leqq 0 .
$$

If $u^{*}(\cdot, 0) \leqq v_{*}(\cdot, 0)$ in $\bar{\Omega}$, then $u^{*} \leqq v_{*}$ in $\bar{\Omega} \times(0, T)$.

Proof. To simplify notation we write $u$ for $u^{*}$ and $v$ for $v_{*}$.

1. Doubling the variables. Suppose by contradiction that $M:=u\left(x_{0}, t_{0}\right)-v\left(x_{0}, t_{0}\right)>$ 0 for some $\left(x_{0}, t_{0}\right) \in \bar{\Omega} \times(0, T)$. Define an upper semicontinuous function $\Phi^{\zeta}:(\bar{\Omega} \times[0, T])^{2} \rightarrow$ $\mathbf{R} \cup\{-\infty\}$ by

$$
\Phi^{\zeta}(x, t, y, s):=u(x, t)-v(y, s)-\phi^{\zeta}(x, t, y, s)
$$

with

$$
\begin{aligned}
\phi^{\zeta}(x, t, y, s):=\alpha & \left(\left|x^{\prime}-y^{\prime}\right|^{4}+\left|x_{n}-y_{n}-\zeta\right|^{2}+\left|x_{n}-y_{n}-\zeta+\beta(t-s)\right|^{2}\right) \\
& +\frac{\sigma}{T-t}+\frac{\sigma}{T-s} .
\end{aligned}
$$

If $t=T$ or $s=T$, we conventionally set $\Phi^{\zeta}(x, t, y, s)=-\infty$. Here constants $\alpha \geqq 1, \sigma>0$ and $\zeta \in \mathbf{R}$ are chosen so that

$$
\sigma \leqq \frac{M\left(T-t_{0}\right)}{8}
$$

and

$$
|\zeta|<\kappa(\alpha):=\frac{1}{\alpha^{\gamma}} \min \left\{C_{1}, C_{2}\right\} \quad \text { with } \gamma \geqq 1, C_{1}:=\sqrt{\frac{M}{8}}, C_{2}:=\frac{\sigma}{4 \beta T^{2}} .
$$

Such choices guarantee that

$$
\Phi^{\zeta}\left(x_{0}, t_{0}, x_{0}, t_{0}\right) \geqq \frac{M}{2} .
$$

Indeed, by the definition of $\Phi^{\zeta}$, we have

$$
\Phi^{\zeta}\left(x_{0}, t_{0}, x_{0}, t_{0}\right)=M-\alpha \cdot 2 \zeta^{2}-\frac{2 \sigma}{T-t_{0}} .
$$

Applying (3.2) and the inequality $\zeta^{2}<C_{1}^{2} / \alpha^{2 \gamma}$ coming from (3.3), we see that

$$
\Phi^{\zeta}\left(x_{0}, t_{0}, x_{0}, t_{0}\right) \geqq M-\alpha \cdot 2 \cdot \frac{M}{8 \alpha^{2 \gamma}}-\frac{2}{T-t_{0}} \cdot \frac{M\left(T-t_{0}\right)}{8}=M-\frac{M}{4 \alpha^{2 \gamma-1}}-\frac{M}{4} .
$$

Since $\alpha^{2 \gamma-1} \geqq 1$, this yields (3.4).

Let $\left(\hat{x}^{\zeta}, \hat{t}^{\zeta}, \hat{y}^{\zeta}, \hat{s}^{\zeta}\right)$ be a maximum point of $\Phi^{\zeta}$ over $(\bar{\Omega} \times[0, T])^{2}$. This maximum is attained in a compact set $\left(\overline{B_{R}(0)} \times[0, T]\right)^{2}$ if we choose $R>0$ such that $u-v \leqq M / 4$ in $\left(\bar{\Omega} \backslash B_{R}(0)\right) \times$ $[0, T)$. Existence of such $R$ is guaranteed by (3.1). Note that by (3.4) we have

$$
\Phi^{\zeta}\left(\hat{x}^{\zeta}, \hat{t}^{\zeta}, \hat{y}^{\zeta}, \hat{s}^{\zeta}\right) \geqq \Phi^{\zeta}\left(x_{0}, t_{0}, x_{0}, t_{0}\right) \geqq \frac{M}{2} .
$$


Also, notice that $\left(\hat{x}^{\zeta}, \hat{t}^{\zeta}\right)$ is a maximizer of

$$
(x, t) \mapsto \Phi^{\zeta}\left(x, t, \hat{y}^{\zeta}, \hat{s}^{\zeta}\right)=u(x, t)-v\left(\hat{y}^{\zeta}, \hat{s}^{\zeta}\right)-\phi^{\zeta}\left(x, t, \hat{y}^{\zeta}, \hat{s}^{\zeta}\right)
$$

while $\left(\hat{y}^{\zeta}, \hat{s}^{\zeta}\right)$ is a minimizer of

$$
(y, s) \mapsto-\Phi^{\zeta}\left(\hat{x}^{\zeta}, \hat{t}^{\zeta}, y, s\right)=v(y, s)-u\left(\hat{x}^{\zeta}, \hat{t}^{\zeta}\right)-\left(-\phi^{\zeta}\left(\hat{x}^{\zeta}, \hat{t}^{\zeta}, y, s\right)\right) .
$$

For later use we set $\hat{Z}^{\zeta}:=\left(\hat{x}^{\zeta}, \hat{t}^{\zeta}, \hat{y}^{\zeta}, \hat{s}^{\zeta}\right)$, and we compute the derivatives of $\phi^{\zeta}$ :

$$
\begin{aligned}
\nabla_{x^{\prime}} \phi^{\zeta}(x, t, y, s) & =-\nabla_{y^{\prime}} \phi^{\zeta}(x, t, y, s)=4 \alpha\left|x^{\prime}-y^{\prime}\right|^{2}\left(x^{\prime}-y^{\prime}\right), \\
\phi_{x_{n}}^{\zeta}(x, t, y, s) & =-\phi_{y_{n}}^{\zeta}(x, t, y, s) \\
& =2 \alpha\left\{\left(x_{n}-y_{n}-\zeta\right)+\left(x_{n}-y_{n}-\zeta+\beta(t-s)\right)\right\}, \\
\phi_{t}^{\zeta}(x, t, y, s) & =2 \alpha \beta\left(x_{n}-y_{n}-\zeta+\beta(t-s)\right)+\frac{\sigma}{(T-t)^{2}}, \\
\phi_{s}^{\zeta}(x, t, y, s) & =-2 \alpha \beta\left(x_{n}-y_{n}-\zeta+\beta(t-s)\right)+\frac{\sigma}{(T-s)^{2}} .
\end{aligned}
$$

In particular,

$$
\phi_{t}^{\zeta}(x, t, y, s)+\phi_{s}^{\zeta}(x, t, y, s)=\frac{\sigma}{(T-t)^{2}}+\frac{\sigma}{(T-s)^{2}} \geqq \frac{\sigma}{T^{2}}+\frac{\sigma}{T^{2}}=\frac{2 \sigma}{T^{2}} .
$$

2. Estimates for the maximum points $\hat{Z}^{\zeta}$. Set $N:=\sup _{\bar{\Omega} \times[0, T)} u+\sup _{\bar{\Omega} \times[0, T)}(-v)$. Then, by (3.5), we have $0 \leqq \Phi^{\zeta}\left(\hat{Z}^{\zeta}\right) \leqq N-\phi^{\zeta}\left(\hat{Z}^{\zeta}\right)$ and this implies that

$$
\alpha\left|\hat{x}^{\prime \zeta}-\hat{y}^{\zeta \zeta}\right|^{4} \leqq N, \alpha\left|\hat{x}_{n}^{\zeta}-\hat{y}_{n}^{\zeta}-\zeta\right|^{2} \leqq N, \alpha\left|\hat{x}_{n}^{\zeta}-\hat{y}_{n}^{\zeta}-\zeta+\beta\left(\hat{t}^{\zeta}-\hat{s}^{\zeta}\right)\right|^{2} \leqq N
$$

where we write $\hat{x}^{\zeta}=\left(\hat{x}^{\prime \zeta}, \hat{x}_{n}^{\zeta}\right)$ and $\hat{y}^{\zeta}=\left(\hat{y}^{\prime \zeta}, \hat{y}_{n}^{\zeta}\right)$. Using an elemental inequality $(a-b)^{2} \leqq$ $2\left(a^{2}+b^{2}\right)$ for $a, b \in \mathbf{R}$, we further observe

$$
\begin{aligned}
\alpha \beta^{2}\left(\hat{t}^{\zeta}-\hat{s}^{\zeta}\right)^{2} & =\alpha\left\{\left(\hat{x}_{n}^{\zeta}-\hat{y}_{n}^{\zeta}-\zeta+\beta\left(\hat{t}^{\zeta}-\hat{s}^{\zeta}\right)\right)-\left(\hat{x}_{n}^{\zeta}-\hat{y}_{n}^{\zeta}-\zeta\right)\right\}^{2} \\
& \leqq 2 \alpha\left\{\left(\hat{x}_{n}^{\zeta}-\hat{y}_{n}^{\zeta}-\zeta+\beta\left(\hat{t}^{\zeta}-\hat{s}^{\zeta}\right)\right)^{2}+\left(\hat{x}_{n}^{\zeta}-\hat{y}_{n}^{\zeta}-\zeta\right)^{2}\right\} \leqq 4 N .
\end{aligned}
$$

We next show that $\hat{t}^{\zeta}$ and $\hat{s}^{\zeta}$ are uniformly away from $T$. In fact, since $(u-v)\left(\hat{Z}^{\zeta}\right) \leqq N$ and $\phi^{\zeta}\left(\hat{Z}^{\zeta}\right) \geqq \sigma /\left(T-\hat{t}^{\zeta}\right),(3.5)$ implies

$$
\frac{M}{2} \leqq N-\frac{\sigma}{T-\hat{t}^{\zeta}}
$$

Arranging this inequality shows

$$
\hat{t}^{\zeta} \leqq T-\frac{\sigma}{N-(M / 2)}=: T^{-} .
$$

In the same manner we obtain $\hat{s}^{\zeta} \leqq T^{-}$.

It also turns out that neither $\hat{t}^{\zeta}$ nor $\hat{s}^{\zeta}$ lies on the initial time. Namely, we prove that

$$
\exists \alpha_{0}>0, \forall \alpha \geqq \alpha_{0}, \forall|\zeta|<\kappa(\alpha), \hat{t}^{\zeta}>0 \text { and } \hat{s}^{\zeta}>0 .
$$


Suppose the contrary; then there would exist a sequence $\left\{\alpha_{j}\right\}_{j=1}^{\infty} \subset(0, \infty)$ such that $\alpha_{j} \rightarrow \infty$ as $j \rightarrow \infty$ and

$$
\exists\left|\zeta_{j}\right|<\kappa\left(\alpha_{j}\right), \hat{t}^{\zeta_{j}}=0 \text { or } \hat{s}^{\zeta_{j}}=0
$$

Since $\hat{x}^{\zeta_{j}}, \hat{y}^{\zeta_{j}} \in \overline{B_{R}(0)}$ and $\hat{t}^{\zeta_{j}}, \hat{s}^{\zeta_{j}} \in\left[0, T^{-}\right]$, we may assume that the sequence $\left\{\left(\hat{x}^{\zeta_{j}}, \hat{t}^{\zeta_{j}}, \hat{y}^{\zeta_{j}}, \hat{s}^{\zeta_{j}}\right)\right\}_{j=1}^{\infty}$ converges to some $(\bar{x}, \bar{t}, \bar{y}, \bar{s})$ as $j \rightarrow \infty$ with $\bar{x}, \bar{y} \in \overline{B_{R}(0)}$ and $\bar{t}, \bar{s} \in\left[0, T^{-}\right]$. Taking the limit along $\left\{\alpha_{j}\right\}_{j=1}^{\infty}$ and $\left\{\zeta_{j}\right\}_{j=1}^{\infty}$ in (3.9), we see that the first and the second inequality there imply $\bar{x}^{\prime}=\bar{y}^{\prime}$ and $\bar{x}_{n}=\bar{y}_{n}$ respectively. Here we have used the fact that $\zeta_{j} \rightarrow 0$ as $j \rightarrow \infty$, which comes from (3.3). We thus have $\bar{x}=\bar{y}$. Similarly, (3.10) shows $\bar{t}=\bar{s}$, and from this it follows that $\bar{t}=\bar{s}=0$ because of (3.12).

Now, in view of (3.5) we have

$$
\frac{M}{2} \leqq \Phi^{\zeta_{j}}\left(\hat{x}^{\zeta_{j}}, \hat{t}^{\zeta_{j}}, \hat{y}^{\zeta_{j}}, \hat{s}^{\zeta_{j}}\right) \leqq u\left(\hat{x}^{\zeta_{j}}, \hat{t}^{\zeta_{j}}\right)-v\left(\hat{y}^{\zeta_{j}}, \hat{s}^{\zeta_{j}}\right)
$$

By the upper semicontinuity of $u$ and the lower semicontinuity of $v$, sending $j \rightarrow \infty$ implies $M / 2 \leqq u(\bar{x}, \bar{t})-v(\bar{x}, \bar{t})$. Since $\bar{t}=0$, this contradicts the initial condition, and hence (3.11) follows. Hereafter we fix $\alpha$ such that $\alpha \geqq \alpha_{0}$. (We do not take the limit of $\alpha$ in the following argument.)

3. Violation of the boundary condition. We show that, if the maximum point lies on the boundary, then the equation (1.1) should be satisfied by $\phi^{\zeta}$. First, we compute

$$
\begin{aligned}
\phi_{t}^{\zeta}(x, t, y, s)-\beta \phi_{x_{n}}^{\zeta}(x, t, y, s) & =\frac{\sigma}{(T-t)^{2}}-2 \alpha \beta\left(x_{n}-y_{n}-\zeta\right) \\
& \geqq \frac{\sigma}{T^{2}}-2 \alpha \beta\left(x_{n}-y_{n}-\zeta\right), \\
-\phi_{s}^{\zeta}(x, t, y, s)-\beta\left(-\phi_{y_{n}}^{\zeta}(x, t, y, s)\right) & =-\frac{\sigma}{(T-s)^{2}}-2 \alpha \beta\left(x_{n}-y_{n}-\zeta\right) \\
& \leqq-\frac{\sigma}{T^{2}}-2 \alpha \beta\left(x_{n}-y_{n}-\zeta\right) .
\end{aligned}
$$

Let us study the maximum point $\left(\hat{x}^{\zeta}, \hat{t}^{\zeta}\right)$ of the map (3.6). Suppose that $\hat{x}_{n}^{\zeta}=0$. Then

$$
\phi_{t}^{\zeta}\left(\hat{Z}^{\zeta}\right)-\beta \phi_{x_{n}}^{\zeta}\left(\hat{Z}^{\zeta}\right) \geqq \frac{\sigma}{T^{2}}+2 \alpha \beta\left(\hat{y}_{n}^{\zeta}+\zeta\right)
$$

Noting that $\hat{y}_{n}^{\zeta}+\zeta \geqq 0-\kappa(\alpha) \geqq-C_{2} / \alpha^{\gamma}$ and that $\alpha^{\gamma-1} \geqq 1$, we estimate

$$
\begin{aligned}
\phi_{t}^{\zeta}\left(\hat{Z}^{\zeta}\right)-\beta \phi_{x_{n}}^{\zeta}\left(\hat{Z}^{\zeta}\right) & \geqq \frac{\sigma}{T^{2}}-2 \alpha^{1-\gamma} \beta C_{2} \geqq \frac{\sigma}{T^{2}}-2 \beta \cdot \frac{\sigma}{4 \beta T^{2}} \\
& =\frac{\sigma}{T^{2}}-\frac{\sigma}{2 T^{2}}=\frac{\sigma}{2 T^{2}}>0
\end{aligned}
$$

which implies that the boundary condition (1.2) is violated. A similar conclusion is obtained for the minimum point $\left(\hat{y}^{\zeta}, \hat{s}^{\zeta}\right)$ of the map (3.7). Indeed, if $\hat{y}_{n}^{\zeta}=0$, we have

$$
-\phi_{s}^{\zeta}\left(\hat{Z}^{\zeta}\right)-\beta\left(-\phi_{y_{n}}^{\zeta}\left(\hat{Z}^{\zeta}\right)\right) \leqq-\frac{\sigma}{T^{2}}-2 \alpha \beta\left(\hat{x}_{n}^{\zeta}-\zeta\right)
$$


Applying $\hat{x}_{n}^{\zeta}-\zeta \geqq 0-\kappa(\alpha) \geqq-C_{2} / \alpha^{\gamma}$ to the above inequality yields violation of the boundary condition.

4. The case $\nabla_{x} \phi^{\zeta}\left(\hat{Z}^{\zeta}\right) \neq 0$ for some $|\zeta|<\kappa(\alpha)$. In this case the argument is classical. Making use of Crandall-Ishii lemma ([10, Theorem 3.2, Theorem 8.3]), we see that there exist $X^{\zeta}, Y^{\zeta} \in \mathbf{S}^{n}$ such that

$$
\begin{aligned}
\left(\left(\nabla_{x} \phi^{\zeta}\left(\hat{Z}^{\zeta}\right), \phi_{t}^{\zeta}\left(\hat{Z}^{\zeta}\right)\right), X^{\zeta}\right) & \in \overline{\mathcal{P}}^{2,+} u\left(\hat{x}^{\zeta}, \hat{t}^{\zeta}\right), \\
\left(\left(-\nabla_{y} \phi^{\zeta}\left(\hat{Z}^{\zeta}\right),-\phi_{s}^{\zeta}\left(\hat{Z}^{\zeta}\right)\right),-Y^{\zeta}\right) & \in \overline{\mathcal{P}}^{2,-} v\left(\hat{y}^{\zeta}, \hat{s}^{\zeta}\right), \\
\left(\begin{array}{cc}
X^{\zeta} & O \\
O & Y^{\zeta}
\end{array}\right) & \leqq A+A^{2}
\end{aligned}
$$

with

$$
A=\nabla_{(x, y)}^{2} \phi^{\zeta}\left(\hat{Z}^{\zeta}\right)=\left(\begin{array}{cc}
B & -B \\
-B & B
\end{array}\right), \quad B=\nabla_{x}^{2} \phi^{\zeta}\left(\hat{Z}^{\zeta}\right)
$$

Since $A$ is of the above form, operating $(\xi, \xi) \in \mathbf{R}^{n} \times \mathbf{R}^{n}$ to (3.15) implies that $X^{\zeta}+Y^{\zeta} \leqq O$. Set $p^{\zeta}:=\nabla_{x} \phi^{\zeta}\left(\hat{Z}^{\zeta}\right)=-\nabla_{y} \phi^{\zeta}\left(\hat{Z}^{\zeta}\right) \neq 0$. By the fact that the boundary condition (1.2) breaks for $\phi^{\zeta}$ and Remark 2.2, we derive from (3.13) and (3.14)

$$
\phi_{t}^{\zeta}\left(\hat{Z}^{\zeta}\right)+F\left(p^{\zeta}, X^{\zeta}\right) \leqq 0, \quad-\phi_{s}^{\zeta}\left(\hat{Z}^{\zeta}\right)+F\left(p^{\zeta},-Y^{\zeta}\right) \geqq 0
$$

By (F2), the ellipticity of $F$, and (3.8), subtraction of these inequalities implies

$$
0 \geqq \phi_{t}^{\zeta}\left(\hat{Z}^{\zeta}\right)+\phi_{s}^{\zeta}\left(\hat{Z}^{\zeta}\right)+F\left(p^{\zeta}, X^{\zeta}\right)-F\left(p^{\zeta},-Y^{\zeta}\right) \geqq \frac{2 \sigma}{T^{2}}+0>0
$$

This is a contradiction.

5. The case $\nabla_{x} \phi^{\zeta}\left(\hat{Z}^{\zeta}\right)=0$ for all $|\zeta|<\kappa(\alpha)$. The constancy lemma (Lemma 2.3) is able to be applied in this case. We apply the lemma for

$$
\begin{aligned}
& N=2 n+2, d=1, K=\left(\overline{B_{R}(0)} \times[0, T]\right)^{2}, G=(-\kappa(\alpha), \kappa(\alpha)), \\
& r=x_{n}, \rho=\left(x^{\prime}, t, y, s\right), h(r, \rho)=u(x, t)-v(y, s), \phi(r, \rho)=\phi^{\zeta}(x, t, y, s)
\end{aligned}
$$

to see that

$$
\Phi^{0}\left(\hat{Z}^{0}\right)=\Phi^{\zeta}\left(\hat{Z}^{\zeta}\right) \text { for all }|\zeta|<\kappa(\alpha)
$$

Also, by the fact $\nabla_{x} \phi^{\zeta}\left(\hat{Z}^{\zeta}\right)=0$ and the derivatives of $\phi^{\zeta}$ computed in Step 1, we have for all $|\zeta|<\kappa(\alpha)$

$$
\hat{x}^{\prime \zeta}=\hat{y}^{\prime \zeta}
$$

and

$$
\left(\hat{x}_{n}^{\zeta}-\hat{y}_{n}^{\zeta}-\zeta\right)+\left(\hat{x}_{n}^{\zeta}-\hat{y}_{n}^{\zeta}-\zeta+\beta\left(\hat{t}^{\zeta}-\hat{s}^{\zeta}\right)\right)=0 .
$$

The latter one is equivalent to

$$
\hat{x}_{n}^{\zeta}-\hat{y}_{n}^{\zeta}-\zeta=-\frac{\beta}{2}\left(\hat{t}^{\zeta}-\hat{s}^{\zeta}\right)
$$


and in particular

$$
\hat{x}_{n}^{0}-\hat{y}_{n}^{0}=-\frac{\beta}{2}\left(\hat{t}^{0}-\hat{s}^{0}\right) .
$$

We now define a compact set $U \subset(\bar{\Omega} \times[0, T])^{2}$ by

$$
U:=\left\{(x, t, y, s) \in\left(\overline{B_{R}(0)} \times[0, T]\right)^{2}|| x_{n}-y_{n}+\frac{\beta}{2}(t-s) \mid \leqq \frac{\kappa(\alpha)}{2}\right\}
$$

and an upper semicontinuous function $\Theta:(\bar{\Omega} \times[0, T])^{2} \rightarrow \mathbf{R} \cup\{-\infty\}$ by

$$
\Theta(x, t, y, s):=u(x, t)-v(y, s)-\rho(x, t, y, s)
$$

with

$$
\rho(x, t, y, s):=\alpha\left(\left|x^{\prime}-y^{\prime}\right|^{4}+\frac{\beta^{2}}{2}(t-s)^{2}\right)+\frac{\sigma}{T-t}+\frac{\sigma}{T-s} .
$$

For these we assert that

$$
\max _{U} \Theta=\Theta\left(\hat{Z}^{0}\right)
$$

Since $\hat{x}^{0}, \hat{y}^{0} \in \overline{B_{R}(0)}, \hat{t}^{0}, \hat{s}^{0} \in(0, T)$ and $\hat{x}_{n}^{0}-\hat{y}_{n}^{0}+\beta\left(\hat{t}^{0}-\hat{s}^{0}\right) / 2=0$ by (3.19), we see that $\hat{Z}^{0}$ belongs to the interior of $U$. Thus $\max _{U} \Theta \geqq \Theta\left(\hat{Z}^{0}\right)$. To prove the opposite inequality, let us fix $(x, t, y, s) \in U$. We set $\tilde{\zeta}:=x_{n}-y_{n}+\beta(t-s) / 2$; then $|\tilde{\zeta}| \leqq \kappa(\alpha) / 2$ and an easy computation shows that

$$
\phi^{\tilde{\zeta}}(x, t, y, s)=\alpha\left(\left|x^{\prime}-y^{\prime}\right|^{4}+\frac{\beta^{2}}{2}(t-s)^{2}\right)+\frac{\sigma}{T-t}+\frac{\sigma}{T-s}=\rho(x, t, y, s) .
$$

Therefore

$$
\Theta(x, t, y, s)=u(x, t)-v(y, s)-\phi^{\tilde{\zeta}}(x, t, y, s)=\Phi^{\tilde{\zeta}}(x, t, y, s) \leqq \Phi^{\tilde{\zeta}}\left(\hat{Z}^{\tilde{\zeta}}\right) .
$$

We now apply (3.16) to obtain

$$
\Theta(x, t, y, s) \leqq \Phi^{0}\left(\hat{Z}^{0}\right)
$$

At $\hat{Z}^{0}$ the number $\tilde{\zeta}$ defined above is 0 by virtue of (3.19). This and (3.21) imply that $\phi^{0}\left(\hat{Z}^{0}\right)=\rho\left(\hat{Z}^{0}\right)$, and so $\Phi^{0}\left(\hat{Z}^{0}\right)=\Theta\left(\hat{Z}^{0}\right)$. Consequently,

$$
\Theta(x, t, y, s) \leqq \Theta\left(\hat{Z}^{0}\right) .
$$

(3.20) is proved.

Now we list the derivatives of the test function $\rho$, which are

$$
\begin{aligned}
\nabla_{x^{\prime}} \rho(x, t, y, s) & =-\nabla_{y^{\prime}} \rho(x, t, y, s)=4 \alpha\left|x^{\prime}-y^{\prime}\right|^{2}\left(x^{\prime}-y^{\prime}\right), \\
\rho_{x_{n}}(x, t, y, s) & =\rho_{y_{n}}(x, t, y, s)=0 \\
\rho_{t}(x, t, y, s) & =\alpha \beta^{2}(t-s)+\frac{\sigma}{(T-t)^{2}}, \\
\rho_{s}(x, t, y, s) & =-\alpha \beta^{2}(t-s)+\frac{\sigma}{(T-s)^{2}}, \\
\nabla_{x^{\prime}}^{2} \rho(x, t, y, s) & =4 \alpha\left(2\left(x^{\prime}-y^{\prime}\right) \otimes\left(x^{\prime}-y^{\prime}\right)+\left|x^{\prime}-y^{\prime}\right|^{2} I\right), \\
\rho_{x_{n} x_{i}}(x, t, y, s) & =0 .
\end{aligned}
$$


Similarly to Step 3, the boundary condition is not achieved by the test function $\rho$ at the maximum point. In fact, if $\hat{x}_{n}^{0}=0$, by using (3.19) we observe

$$
\begin{aligned}
\rho_{t}\left(\hat{Z}^{0}\right)-\beta \rho_{x_{n}}\left(\hat{Z}^{0}\right) & =\left\{\alpha \beta^{2}\left(\hat{t}^{0}-\hat{s}^{0}\right)+\frac{\sigma}{\left(T-\hat{t}^{0}\right)^{2}}\right\}-0 \\
& =2 \alpha \beta \hat{y}_{n}^{0}+\frac{\sigma}{\left(T-\hat{t}^{0}\right)^{2}} \geqq \frac{\sigma}{T^{2}}>0 .
\end{aligned}
$$

In the same manner, it follows that

$$
-\rho_{s}\left(\hat{Z}^{0}\right)-\beta\left(-\rho_{y_{n}}\left(\hat{Z}^{0}\right)\right) \leqq-\frac{\sigma}{T^{2}}<0 .
$$

By (3.17) the spatial derivatives of $\rho$ at $\hat{Z}^{0}$ are now

$$
\nabla_{x} \rho\left(\hat{Z}^{0}\right)=\nabla_{y} \rho\left(\hat{Z}^{0}\right)=0, \quad \nabla_{x}^{2} \rho\left(\hat{Z}^{0}\right)=\nabla_{y}^{2} \rho\left(\hat{Z}^{0}\right)=O .
$$

Therefore we have the viscosity inequalities

$$
\rho_{t}\left(\hat{Z}^{0}\right)+F_{*}(0, O) \leqq 0, \quad-\rho_{s}\left(\hat{Z}^{0}\right)+F^{*}(0, O) \geqq 0
$$

(Here we do not need to apply Crandall-Ishii lemma.) Combining these and using (F3), we get

$$
0 \geqq \rho_{t}\left(\hat{Z}^{0}\right)+\rho_{s}\left(\hat{Z}^{0}\right)=\frac{\sigma}{\left(T-\hat{t}^{0}\right)^{2}}+\frac{\sigma}{\left(T-\hat{s}^{0}\right)^{2}} \geqq \frac{\sigma}{T^{2}}+\frac{\sigma}{T^{2}}>0,
$$

which is a contradiction.

Remark 3.2. In the proof of Theorem 3.1, the positivity of $\beta$ is needed to guarantee that $\hat{t}^{\zeta}$ and $\hat{s}^{\zeta}$ converge to the same value, which is used to prove (3.11). When $\beta=0$, this may not hold since the left-hand side of (3.10) is 0 . In fact, the comparison principle fails for semicontinuous viscosity solutions of (DB) with $\beta=0$ even if the equation is non-singular and first order ([2, Example A.5]).

In contrast, our existence results (Theorem 4.5 and Theorem 4.11) presented in the next section hold even if $\beta=0$. See Remark 4.6.

Remark 3.3. For $a \in \mathbf{R}$ we define

$$
\begin{aligned}
& K_{a}^{+}(\bar{\Omega} \times[0, T)) \\
& :=\left\{u: \bar{\Omega} \times[0, T) \rightarrow \mathbf{R} \mid \begin{array}{c}
u \text { is bounded from below; } \\
\quad: a \text { in }\left(\bar{\Omega} \backslash B_{R}(0)\right) \times[0, T) \text { for some } R>0
\end{array}\right\}, \\
& K_{a}^{-}(\bar{\Omega} \times[0, T)) \\
& :=\left\{u: \bar{\Omega} \times[0, T) \rightarrow \mathbf{R} \mid \begin{array}{c}
u \text { is bounded from above; } \\
u \leqq a \text { in }\left(\bar{\Omega} \backslash B_{R}(0)\right) \times[0, T) \text { for some } R>0
\end{array}\right\} .
\end{aligned}
$$

The comparison principle (Theorem 3.1) is then applicable to a subsolution $u \in K_{a}^{-}(\bar{\Omega} \times$ $[0, T))$ and a supersolution $v \in K_{a}^{+}(\bar{\Omega} \times[0, T))$ since $(3.1)$ is satisfied. 
We now state a uniqueness result. Define

$$
\begin{aligned}
& K_{a}(\bar{\Omega} \times[0, T)) \\
& :=K_{a}^{-}(\bar{\Omega} \times[0, T)) \cap K_{a}^{+}(\bar{\Omega} \times[0, T)) \\
& =\left\{u: \bar{\Omega} \times[0, T) \rightarrow \mathbf{R} \mid \begin{array}{c}
u \text { is bounded; } \\
u=a \text { in }\left(\bar{\Omega} \backslash B_{R}(0)\right) \times[0, T) \text { for some } R>0
\end{array}\right\}
\end{aligned}
$$

and

$$
C_{a}(\bar{\Omega}):=\left\{u_{0} \in C(\bar{\Omega}) \mid u_{0} \equiv a \text { in } \bar{\Omega} \backslash B_{R}(0) \text { for some } R>0\right\} .
$$

Theorem 3.4 (Uniqueness). Assume $u_{0} \in C_{a}(\bar{\Omega})$. If $u, v \in K_{a}(\bar{\Omega} \times[0, T))$ are viscosity solutions of $(\mathrm{DB})$, then $u=v$ and they are continuous in $\bar{\Omega} \times[0, T)$.

Proof. Since $u$ is a subsolution and $v$ is a supersolution, Theorem 3.1 and Remark 3.3 imply that $u^{*} \leqq v_{*}$. Similarly, we have $v^{*} \leqq u_{*}$. Combining these inequalities, we obtain $u^{*} \leqq v_{*} \leqq v^{*} \leqq u_{*} \leqq u^{*}$ in $\bar{\Omega} \times[0, T)$. Thus all of the inequalities should be equalities, which gives the results.

Remark 3.5. The same choice of the test function $\Phi^{\zeta}$ works for a domain $\Omega$ of layer type: $\Omega=\left\{x=\left(x^{\prime}, x_{n}\right) \in \mathbf{R}^{n-1} \times \mathbf{R} \mid 0<x_{n}<L\right\}$ with $L>0$. The comparison principle is proven in the same argument.

\section{Existence of solutions}

\subsection{Stability and consistency}

We first prepare stability and consistency results for viscosity solutions.

Proposition 4.1 (Stability). Let $\mathcal{S}$ be a family of viscosity subsolutions (resp. viscosity supersolutions) of (1.1) and (1.2). Set $u(x, t):=\sup \{w(x, t) \mid w \in \mathcal{S}\} \quad$ (resp. $u(x, t):=$ $\inf \{w(x, t) \mid w \in \mathcal{S}\})$ and assume that $u^{*}<\infty$ (resp. $\left.u_{*}>-\infty\right)$ in $\bar{\Omega} \times(0, T)$. Then $u$ is a viscosity subsolution (resp. a viscosity supersolution) of (1.1) and (1.2).

For the proof see, e.g., [10, Lemma 4.2] and [29, Lemma 2.4.1].

Proposition 4.2 (Consistency). Assume that $u \in C^{2,1}(\bar{\Omega} \times(0, T))$ is a classical subsolution (resp. a classical supersolution) of (1.1) and (1.2), i.e.,

$$
\begin{cases}u_{t}(x, t)+F_{*}\left(\nabla u(x, t), \nabla^{2} u(x, t)\right) \leqq 0 & \text { in } \Omega \times(0, T), \\ \left(\text { resp. } u_{t}(x, t)+F^{*}\left(\nabla u(x, t), \nabla^{2} u(x, t)\right) \geqq 0\right) & \\ u_{t}(x, t)-\beta u_{x_{n}}(x, t) \leqq 0 \quad(\text { resp. } \geqq 0) & \text { on } \partial \Omega \times(0, T) .\end{cases}
$$

Then $u$ is a viscosity subsolution (resp. a viscosity supersolution) of (1.1) and (1.2). 
Proof. Let $((p, \tau), X) \in \mathcal{P}^{2,+} u\left(x_{0}, t_{0}\right)$ and take $\phi \in C^{2,1}(\bar{\Omega} \times(0, T))$ such that $u-\phi$ attains a maximum at $\left(x_{0}, t_{0}\right)$ and $((p, \tau), X)=\left(\left(\nabla \phi, \phi_{t}\right), \nabla^{2} \phi\right)$ at $\left(x_{0}, t_{0}\right)$. If $x_{0}>0$, it follows from a maximum principle for smooth functions that $\left(\nabla u, u_{t}\right)=\left(\nabla \phi, \phi_{t}\right)$ and $\nabla^{2} u \leqq \nabla^{2} \phi$ at $\left(x_{0}, t_{0}\right)$. This together with degenerate ellipticity of $F_{*}$ shows that

$$
\tau+F_{*}(p, X) \leqq u_{t}\left(x_{0}, t_{0}\right)+F_{*}\left(\nabla u\left(x_{0}, t_{0}\right), \nabla^{2} u\left(x_{0}, t_{0}\right)\right) \leqq 0 .
$$

If $x_{0}=0$, we have $u_{t}=\phi_{t}$ and $u_{x_{n}} \leqq \phi_{x_{n}}$ at $\left(x_{0}, t_{0}\right)$. Therefore $\tau-\beta p_{n} \leqq u_{t}\left(x_{0}, t_{0}\right)-$ $\beta u_{x_{n}}\left(x_{0}, t_{0}\right) \leqq 0$.

\subsection{Uniformly continuous initial data}

We prove that there is a viscosity solution of (DB) when the initial data $u_{0}$ is uniformly continuous in $\bar{\Omega}$, i.e, $u_{0} \in U C(\bar{\Omega})$. The solution will be constructed by Perron's method ([10, Section 4], [29, Chapter 2.4]), which was originally established in [37]. A unique existence result will be established in the next subsection for initial data being constant at infinity when $F$ is a geometric operator.

In what follows we assume a local boundedness of $F$ :

$$
\mu\left(C_{1}, C_{2}\right):=\sup \left\{|F(p, X)||0<| p \mid \leqq C_{1},\|X\| \leqq C_{2}\right\}<\infty \text { for all } C_{1}, C_{2}>0 .
$$

Note that (F4) implies that $\left|F_{*}(p, X)\right|,\left|F^{*}(p, X)\right| \leqq \mu\left(C_{1}, C_{2}\right)$ if $|p| \leqq C_{1}$ and $\|X\| \leqq C_{2}$.

Let $u_{0} \in U C(\bar{\Omega})$. Let $\omega_{0}:[0, \infty) \rightarrow[0, \infty)$ be a modulus of continuity of $u_{0}$, which is defined as

$$
\omega_{0}(r):=\sup \left\{\left|u_{0}(x)-u_{0}(y)\right||x, y \in \bar{\Omega},| x-y \mid \leqq r\right\} .
$$

Since $u_{0}$ is uniformly continuous, the modulus $\omega_{0}$ has at most linear growth and satisfies $0=\omega_{0}(0)=\lim _{r \rightarrow 0} \omega_{0}(r)$.

We now take an increasing function $f:[0, \infty) \rightarrow[0, \infty)$ such that $f(0)=0$ and $\lim _{r \rightarrow 0} f(r)=\infty$. By the properties of $\omega_{0}$, we then see that for every $\varepsilon>0$ there is a constant $C_{\varepsilon}>0$ such that

$$
\omega_{0}(r) \leqq \varepsilon+C_{\varepsilon} f(r) \quad \text { for all } r \geqq 0 .
$$

For later use, we further require $f$ to satisfy $f \in C^{2}([0, \infty)), f^{\prime}(0)=f^{\prime \prime}(0)=0, f^{\prime}(r)>0$ for $r>0,\left\|f^{\prime} / r\right\|_{\infty}=\sup _{r \in(0, \infty)}\left|f^{\prime}(r) / r\right|<\infty,\left\|f^{\prime}\right\|_{\infty}<\infty$ and $\left\|f^{\prime \prime}\right\|_{\infty}<\infty$. We denote by $\mathcal{F}$ the set of $f:[0, \infty) \rightarrow[0, \infty)$ satisfying all the conditions above. Examples of $f \in \mathcal{F}$ include $f(r)=\left(r^{4}+1\right)^{1 / 4}-1$ and $f(r)=r-\arctan r$.

For a fixed $y \in \bar{\Omega}$ and $\varepsilon>0$, let us define

$$
\begin{aligned}
V_{y, \varepsilon}^{-}(x, t) & :=u_{0}(y)-\varepsilon-C_{\varepsilon} f(|x-y|)-M_{\varepsilon} t, \\
V_{y, \varepsilon}^{+}(x, t) & :=u_{0}(y)+\varepsilon+C_{\varepsilon} f(|x-y|)+M_{\varepsilon} t
\end{aligned}
$$

where $M_{\varepsilon} \geqq 0$ is a constant given by

$$
M_{\varepsilon}:=\mu\left(C_{\varepsilon}\left\|f^{\prime}\right\|_{\infty}, C_{\varepsilon}\left(\left\|f^{\prime \prime}\right\|_{\infty}+\left\|f^{\prime} / r\right\|_{\infty}\right)\right) .
$$


Since $f$ is now supposed to satisfy $f \in C^{2}([0, \infty))$ and $f^{\prime}(0)=f^{\prime \prime}(0)=0$, it follows that $V_{y, \varepsilon}^{ \pm} \in C^{2,1}(\bar{\Omega} \times[0, T))$. In particular, we have

$$
\nabla V_{y, \varepsilon}^{ \pm}(y, t)=0, \quad \nabla^{2} V_{y, \varepsilon}^{ \pm}(y, t)=O
$$

Also, the definition of $M_{\varepsilon},(4.5)$, gives

$$
-M_{\varepsilon} \leqq F_{*}(0, O) \leqq F^{*}(0, O) \leqq M_{\varepsilon} .
$$

We shall prove that $V_{y, \varepsilon}^{ \pm}$are a classical sub-/supersolution of (DB).

Lemma 4.3. Assume $u_{0} \in U C(\bar{\Omega})$. Assume (F4). Then the functions $V_{y, \varepsilon}^{-}$and $V_{y, \varepsilon}^{+}$given in (4.3) and (4.4) are, respectively, a classical subsolution and a classical supersolution of (DB).

Proof. For $(x, t) \in \bar{\Omega} \times(0, T)$, we see by (4.2) and (4.1) that

$$
V_{y, \varepsilon}^{-}(x, t) \leqq u_{0}(y)-\omega_{0}(|x-y|)-M_{\varepsilon} t \leqq u_{0}(x)-M_{\varepsilon} t .
$$

In particular, $V_{y, \varepsilon}^{-}(x, 0) \leqq u_{0}(x)$, and so the condition at the initial time is fulfilled.

Let us compute the derivatives of $V_{y, \varepsilon}^{-}$at $(x, t) \in \bar{\Omega} \times(0, T)$. Clearly, $\partial_{t} V_{y, \varepsilon}^{-}(x, t)=-M_{\varepsilon}$. When $x=y$, it follows from (4.6) and (4.7) that

$$
\partial_{t} V_{y, \varepsilon}^{-}(y, t)+F_{*}\left(\nabla V_{y, \varepsilon}^{-}(y, t), \nabla^{2} V_{y, \varepsilon}^{-}(y, t)\right)=-M_{\varepsilon}+F_{*}(0, O) \leqq 0 .
$$

Next, assume that $z:=x-y \neq 0$. Then

$$
\begin{aligned}
\nabla V_{y, \varepsilon}^{-}(x, t) & =-C_{\varepsilon} f^{\prime}(|z|) \frac{z}{|z|} \neq 0 \\
\nabla^{2} V_{y, \varepsilon}^{-}(x, t) & =-C_{\varepsilon}\left\{f^{\prime \prime}(|z|) \frac{z \otimes z}{|z|^{2}}+\frac{f^{\prime}(|z|)}{|z|}\left(I-\frac{z \otimes z}{|z|^{2}}\right)\right\} .
\end{aligned}
$$

It is well known that $\|\bar{z} \otimes \bar{z}\| \leqq 1$ and $\|I-\bar{z} \otimes \bar{z}\| \leqq 1$ for $\bar{z}:=z /|z|$, and thus we see

$$
\left|\nabla V_{y, \varepsilon}^{-}(x, t)\right| \leqq C_{\varepsilon}\left\|f^{\prime}\right\|_{\infty}, \quad\left\|\nabla^{2} V_{y, \varepsilon}^{-}(x, t)\right\| \leqq C_{\varepsilon}\left(\left\|f^{\prime \prime}\right\|_{\infty}+\left\|f^{\prime} / r\right\|_{\infty}\right) .
$$

This and (4.5) yield

$$
\begin{aligned}
& \partial_{t} V_{y, \varepsilon}^{-}(x, t)+F\left(\nabla V_{y, \varepsilon}^{-}(x, t), \nabla^{2} V_{y, \varepsilon}^{-}(x, t)\right) \\
& =-M_{\varepsilon}+F\left(\nabla V_{y, \varepsilon}^{-}(x, t), \nabla^{2} V_{y, \varepsilon}^{-}(x, t)\right) \leqq 0,
\end{aligned}
$$

which together with the case where $x=y$ implies that $V_{y, \varepsilon}^{-}$is a subsolution of the equation (1.1).

It remains to check the boundary condition (1.2). When $x_{n}=0$, we observe

$$
\partial_{x_{n}} V_{y, \varepsilon}^{-}(x, t)=-C_{\varepsilon} f^{\prime}(|x-y|) \frac{-y_{n}}{|x-y|} \geqq 0
$$

if $x \neq y$; otherwise $\partial_{x_{n}} V_{y, \varepsilon}^{-}(x, t)=0$. Therefore

$$
\partial_{t} V_{y, \varepsilon}^{-}(x, t)-\beta \partial_{x_{n}} V_{y, \varepsilon}^{-}(x, t)=-M_{\varepsilon}-\beta \partial_{x_{n}} V_{y, \varepsilon}^{-}(x, t) \leqq 0 .
$$

The proof for $V_{y, \varepsilon}^{-}$is now complete, and that for $V_{y, \varepsilon}^{+}$is parallel. 
Making use of $V_{y, \varepsilon}^{ \pm}$, we next create a viscosity sub- and supersolution satisfying the initial condition. Define

$$
\begin{aligned}
& v^{-}(x, t):=\sup \left\{V_{y, \varepsilon}^{-}(x, t) \mid y \in \bar{\Omega}, \varepsilon>0\right\} \\
& v^{+}(x, t):=\inf \left\{V_{y, \varepsilon}^{+}(x, t) \mid y \in \bar{\Omega}, \varepsilon>0\right\}
\end{aligned}
$$

Since (4.8) implies

$$
V_{y, \varepsilon}^{-}(x, t) \leqq u_{0}(x) \text { for all }(x, t) \in \bar{\Omega} \times(0, T)
$$

for every $y \in \bar{\Omega}$ and $\varepsilon>0$, it follows that $v^{-}$is a real-valued function. Similarly, $v^{+}$is real-valued too. We further prepare a function $\alpha:[0, \infty) \rightarrow[0, \infty)$ defined as

$$
\alpha(t):=\inf _{\varepsilon>0}\left(\varepsilon+M_{\varepsilon} t\right)
$$

By definition we see that $\alpha$ is non-decreasing and satisfies $0=\alpha(0)=\lim _{t \rightarrow \infty} \alpha(t)$.

Proposition 4.4. Assume $u_{0} \in U C(\bar{\Omega})$. Assume (F4). Then the functions $v^{-}$and $v^{+}$given in (4.9) and (4.10) are, respectively, a viscosity subsolution and a viscosity supersolution of (DB). Moreover they satisfy

$$
\begin{aligned}
& u_{0}(x)-\alpha(t) \leqq v^{-}(x, t) \leqq u_{0}(x) \quad \text { for all }(x, t) \in \bar{\Omega} \times[0, T), \\
& u_{0}(x) \leqq v^{+}(x, t) \leqq u_{0}(x)+\alpha(t) \quad \text { for all }(x, t) \in \bar{\Omega} \times[0, T) .
\end{aligned}
$$

Proof. Since $V_{y, \varepsilon}^{-}$is a classical subsolution of (DB) by Lemma 4.3, it follows from the consistency (Proposition 4.2) that $V_{y, \varepsilon}^{-}$is a viscosity subsolution of (DB). Thus the stability result (Proposition 4.1) guarantees that $v^{-}$is a viscosity subsolution of (DB).

Let us prove (4.13). Fix $(x, t) \in \bar{\Omega} \times[0, T)$. The inequality $v^{-}(x, t) \leqq u_{0}(x)$ is a direct consequence of (4.11). In order to derive the other inequality, we observe

$$
v^{-}(x, t) \geqq V_{x, \varepsilon}^{-}(x, t)=u_{0}(x)-\varepsilon-M_{\varepsilon} t .
$$

Taking the supremum with respect to $\varepsilon>0$ implies that $v^{-}(x, t) \geqq u_{0}(x)-\alpha(t)$. The same argument proves (4.14).

Finally, we establish an existence of viscosity solutions to (DB) by Perron's method. For this purpose, we set

$$
\mathcal{S}_{0}:=\left\{\begin{array}{l|l}
w \mid \begin{array}{c}
w \text { is a viscosity subsolution of }(\mathrm{DB}) \\
\text { such that } v^{-} \leqq w \leqq v^{+} \text {in } \bar{\Omega} \times[0, T)
\end{array}
\end{array}\right\},
$$

where $v^{ \pm}$are given by (4.9) and (4.10). Note that $\mathcal{S}_{0}$ is not empty since $v^{-} \in \mathcal{S}_{0}$.

Theorem 4.5 (Existence). Assume $u_{0} \in U C(\bar{\Omega})$. Assume $(\mathrm{F} 4)$. Then $u(x, t):=\sup \{w(x, t) \mid$ $\left.w \in \mathcal{S}_{0}\right\}$ is a viscosity solution of (DB). Moreover, it satisfies

$$
u_{0}(x)-\alpha(t) \leqq u(x, t) \leqq u_{0}(x)+\alpha(t) \quad \text { for all }(x, t) \in \bar{\Omega} \times[0, T) .
$$


Proof. By the definition of $u$ we have $v^{-} \leqq u \leqq v^{+}$in $\bar{\Omega} \times[0, T)$. This and the estimates (4.13) and (4.14) give (4.15). In particular, we have $u^{*}(x, 0)=u_{*}(x, 0)=u_{0}$ in $\bar{\Omega}$. The initial condition is thus satisfied.

The fact that $u$ is a viscosity subsolution of (DB) is guaranteed by the stability, Proposition 4.1. If $u$ were not a supersolution, we would have $u\left(x_{0}, t_{0}\right)<w\left(x_{0}, t_{0}\right)$ for some $\left(x_{0}, t_{0}\right) \in \bar{\Omega} \times(0, T)$ and $w \in \mathcal{S}_{0}$ by the classical argument; see [10, Lemma 4.4] and [29, Lemma 2.4.2] for more details. This contradicts a maximality of $u$.

Remark 4.6. Theorem 4.5 is still true if $\beta=0$. If fact, since $\partial_{t} V_{y, \varepsilon}^{-} \leqq 0 \leqq \partial_{t} V_{y, \varepsilon}^{+}$, we see that $V_{y, \varepsilon}^{-}$and $V_{y, \varepsilon}^{+}$are, respectively, a classical subsolution and a classical supersolution of (1.1) and (1.2) with $\beta=0$. Since Proposition 4.2 (consistency) also holds for $\beta=0$, the same proof by Perron's method remains valid. For the same reason, we obtain Theorem 4.11 in the next subsection even if $\beta=0$.

\subsection{Initial data being constant at infinity}

We next establish a unique existence result of viscosity solutions of (DB) in the class $K_{a}(\bar{\Omega} \times$ $[0, T)$ ) when $u_{0} \in C_{a}(\bar{\Omega})$. Recall that these function spaces are defined before Theorem 3.4.

For this purpose, we assume in this subsection that $F$ is geometric. Namely,

(F5) $F(\lambda p, \lambda X+\sigma p \otimes p)=\lambda F(p, X)$ for all $(p, X) \in\left(\mathbf{R}^{n} \backslash\{0\}\right) \times \mathbf{S}^{n}, \lambda>0$ and $\sigma \in \mathbf{R}$.

The mean curvature flow operator (1.9) satisfies (F5).

Remark 4.7. Assume that $F$ satisfies (F5).

(1) Since we have $F(\lambda p, \lambda X)=\lambda F(p, X)$ by putting $\sigma=0$ in (F5), sending $\lambda \rightarrow 0$ gives

$$
F^{*}(0, O) \geqq \limsup _{\lambda \rightarrow 0} F(\lambda p, \lambda X)=\limsup _{\lambda \rightarrow 0} \lambda F(p, X)=0 \text {. }
$$

Similarly, we have $F_{*}(0, O) \leqq 0$. Thus it follows from (F3) that

$$
F^{*}(0, O)=F_{*}(0, O)=0 \text {. }
$$

(2) It is known that the facts $F^{*}(0, O)=0$ and $F_{*}(0, O)=0$ are, respectively, equivalent to

$$
\sup \{F(p,-I)|0<| p \mid \leqq 1\}<\infty, \inf \{F(p, I)|0<| p \mid \leqq 1\}>-\infty .
$$

See [29, Lemma 1.6.16] for the proofs. For later use, we set

$$
\nu:=\sup \{|F(p, \pm I)||0<| p \mid \leqq 1\}<\infty .
$$

In this subsection, we do not impose the local boundedness (F4) on $F$; instead we use (4.17) to construct barrier functions.

One of important properties of geometric equations is invariance under changes of dependent variables. 
Theorem 4.8 (Invariance). Assume (F5). Let $\theta: \mathbf{R} \rightarrow \mathbf{R}$ be a nondecreasing and upper semicontinuous (resp. lower semicontinuous) function. If $u$ is a viscosity subsolution (resp. supersolution) of (1.1) and (1.2), then so is $\theta \circ u^{*}\left(\right.$ resp. $\left.\theta \circ u_{*}\right)$.

For the proof, see [29, Theorem 4.2.1] for instance. In order to construct a solution in $K_{a}(\bar{\Omega} \times[0, T))$, we reconstruct barriers (sub-/supersolution) without assuming (F4) so that they belong to $K_{a}(\bar{\Omega} \times[0, T))$.

We fix $f \in \mathcal{F}$, where $\mathcal{F}$ is given at the beginning of the previous subsection. Similarly to (4.3) and (4.4), we define

$$
\tilde{V}_{y, \varepsilon}^{ \pm}(x, t):=u_{0}(y) \pm \varepsilon \pm C_{\varepsilon} f(|x-y|) \pm \tilde{M}_{\varepsilon} t
$$

where the only difference is coefficients of $t$. Here $\tilde{M}_{\varepsilon}$ is defined by

$$
\tilde{M}_{\varepsilon}=C_{\varepsilon} \nu \max \left\{\left\|f^{\prime}\right\|_{\infty},\left\|f^{\prime} / r\right\|_{\infty}\right\} .
$$

Lemma 4.9. Assume $u_{0} \in C_{a}(\bar{\Omega})$. Assume (F5). Then the functions $\tilde{V}_{y, \varepsilon}^{-}$and $\tilde{V}_{y, \varepsilon}^{+}$given in (4.18) are, respectively, a classical subsolution and a classical supersolution of (DB).

Proof. We show that $\tilde{V}_{y, \varepsilon}^{-}$is a classical subsolution of $(1.1)$ at $(x, t) \in \Omega \times(0, T)$. The initial condition and the boundary condition can be checked in the same manner as in the proof Lemma 4.3, and so we omit them. We also omit the proof for $\tilde{V}_{y, \varepsilon}^{+}$since it is parallel.

We may suppose that $z:=x-y \neq 0$; otherwise the desired inequality is derived for the same reason as in the proof of Lemma 4.3. Let us set

$$
p=-z, X=-I, \lambda=\frac{C_{\varepsilon} f^{\prime}(|z|)}{|z|}, \sigma=-C_{\varepsilon}\left(\frac{f^{\prime \prime}(|z|)}{|z|^{2}}-\frac{f^{\prime}(|z|)}{|z|^{3}}\right),
$$

so that

$$
\nabla \tilde{V}_{y, \varepsilon}^{-}(x, t)=\lambda p, \quad \nabla^{2} \tilde{V}_{y, \varepsilon}^{-}(x, t)=\lambda X+\sigma p \otimes p .
$$

Thus (F5) implies that

$$
\begin{aligned}
J & :=F\left(\nabla \tilde{V}_{y, \varepsilon}^{-}(x, t), \nabla^{2} \tilde{V}_{y, \varepsilon}^{-}(x, t)\right) \\
& =F(\lambda p, \lambda X+\sigma p \otimes p)=\lambda F(p, X)=\frac{C_{\varepsilon} f^{\prime}(|z|)}{|z|} F(-z,-I) .
\end{aligned}
$$

If $|z| \leqq 1$, we see that $J \leqq C_{\varepsilon}\left\|f^{\prime} / r\right\|_{\infty} \nu \leqq \tilde{M}_{\varepsilon}$. We next let $|z| \geqq 1$. Then $-|z| I \leqq-I$, and so $F(-z,-|z| I) \geqq F(-z,-I)$ by (F2). We therefore have

$$
J \leqq \frac{C_{\varepsilon} f^{\prime}(|z|)}{|z|} F(-z,-|z| I)=C_{\varepsilon} f^{\prime}(|z|) F\left(-\frac{z}{|z|},-I\right) \leqq C_{\varepsilon}\left\|f^{\prime}\right\|_{\infty} \nu \leqq \tilde{M}_{\varepsilon} .
$$

The proof is now complete since $\partial_{t} \tilde{V}_{y, \varepsilon}^{-}(x, t)+J=-\tilde{M}_{\varepsilon}+J \leqq 0$.

For $A^{ \pm} \in \mathbf{R}$ let us set

$$
U^{-}(x, t):=A^{-}+f(|x|)-N t, \quad U^{+}(x, t):=A^{+}-f(|x|)+N t
$$


with $N:=\nu \max \left\{\left\|f^{\prime}\right\|_{\infty},\left\|f^{\prime} / r\right\|_{\infty}\right\}$. In the same manner as in the proof of Lemma 4.9, we see that $U^{-}$and $U^{+}$are, respectively, a classical subsolution and a classical supersolution of (1.1) and (1.2).

Let $u_{0} \in C_{a}(\bar{\Omega})$, and let $R>0$ be a constant such that $u_{0} \equiv a$ in $\bar{\Omega} \backslash B_{R}(0)$. Now, we choose $A>0$ large so that $-A+f(|x|) \leqq u_{0}(x)-a \leqq A-f(|x|)$ for all $x \in B_{R}(0)$. Setting $A^{ \pm}=a \pm A$, we have $U^{-}(x, 0) \leqq u_{0}(x) \leqq U^{+}(x, 0)$ for all $x \in B_{R}(0)$. In order to extend these inequalities to ones in the whole of $\bar{\Omega}$, we define

$$
\tilde{U}^{-}(x, t):=\min \left\{U^{-}(x, t), a\right\}, \quad \tilde{U}^{+}(x, t):=\max \left\{U^{+}(x, t), a\right\} .
$$

It then follows that $\tilde{U}^{-}(\cdot, 0) \leqq u_{0} \leqq \tilde{U}^{+}(\cdot, 0)$ in $\bar{\Omega}$. Moreover, if we set $\theta^{-}(r)=\min \{r, a\}$ and $\theta^{+}(r)=\max \{r, a\}$, then we have $\tilde{U}^{ \pm}=\theta^{ \pm} \circ U^{ \pm}$. Thus Theorem 4.8 implies that $\tilde{U}^{-}$ and $\tilde{U}^{+}$are, respectively, a viscosity subsolution and a viscosity supersolution of (1.1) and (1.2).

We furthermore have $\tilde{U}^{ \pm} \in K_{a}(\bar{\Omega} \times[0, T))$. In fact, for $|x| \geqq R_{0}$ with $R_{0}>0$ satisfying $f\left(R_{0}\right) \geqq A+N T$, we see that $U^{-}(x, t) \geqq a-A+f\left(R_{0}\right)-N T \geqq a$. This shows that $\tilde{U}^{-} \in K_{a}(\bar{\Omega} \times[0, T))$. For the same reason we see that $\tilde{U}^{+} \in K_{a}(\bar{\Omega} \times[0, T))$.

Summarizing the above arguments, we obtain

Proposition 4.10. Assume $u_{0} \in C_{a}(\bar{\Omega})$. Assume (F5). Then, the functions $\tilde{U}^{-}$and $\tilde{U}^{+}$ defined in (4.19) are, respectively, a viscosity subsolution and a viscosity supersolution of (DB). Moreover, $\tilde{U}^{ \pm} \in K_{a}(\bar{\Omega} \times[0, T))$.

A unique existence result immediately follows from this proposition. Set

$$
W^{-}(x, t):=\max \left\{\tilde{U}^{-}(x, t), v^{-}(x, t)\right\}, W^{+}(x, t):=\min \left\{\tilde{U}^{+}(x, t), v^{+}(x, t)\right\},
$$

where $v^{ \pm}$are the functions in (4.9) and (4.10) with $\tilde{V}_{y, \varepsilon}^{ \pm}$instead of $V_{y, \varepsilon}^{ \pm}$. Note that $W^{-}$and $W^{+}$are, respectively, a subsolution and a supersolution of (DB) by Proposition 4.1. Let us define

$$
\mathcal{S}:=\left\{w \mid \begin{array}{c}
w \text { is a viscosity subsolution of }(\mathrm{DB}) \\
\text { such that } W^{-} \leqq w \leqq W^{+} \text {in } \bar{\Omega} \times[0, T)
\end{array}\right\} .
$$

Theorem 4.11 (Unique existence). Assume $u_{0} \in C_{a}(\bar{\Omega})$. Assume (F5). Then $u(x, t):=$ $\sup \{w(x, t) \mid w \in \mathcal{S}\}$ is a unique viscosity solution of (DB) in the class $K_{a}(\bar{\Omega} \times[0, T))$. Moreover, $u \in \underset{\tilde{\Omega}}{C}(\bar{\Omega} \times[0, T))$ and it satisfies (4.15), where in the definition (4.12) of $\alpha$ we replace $M_{\varepsilon}$ by $\tilde{M}_{\varepsilon}$.

Proof. We see that $u$ is a viscosity solution of (DB) and satisfies (4.15) for the same reason as in the proof of Theorem 4.11. Moreover, the fact that $\tilde{U}^{-} \leqq u \leqq \tilde{U}^{+}$gives $u \in K_{a}(\bar{\Omega} \times[0, T))$. The uniqueness and continuity of $u$ is a consequence of Theorem 3.4.

\subsection{Interface evolution equation}

We consider a class of interface evolution equation of the form (1.4) with the dynamic boundary condition (1.10). Set

$$
\mathbf{E}:=\left\{\left(p, Q_{p}(X)\right)\left|p \in \mathbf{R}^{n},\right| p \mid=1, X \in \mathbf{S}^{n}\right\},
$$

where $Q_{p}(X)$ is defined by (1.5). The standard assumption is that $f: \mathbf{E} \rightarrow \mathbf{R}$ is continuous and the equation is parabolic. Namely, 
(f1) $f$ is continuous in $\mathbf{E}$;

(f2) $f\left(p, Q_{p}(X)\right) \geqq f\left(p, Q_{p}(Y)\right)$ for all $p \in \mathbf{R}^{n}$ with $|p|=1$ and $X, Y \in \mathbf{S}^{n}$ such that $Q_{p}(X) \leqq Q_{p}(Y)$.

They imply that the corresponding $F_{f}$ given in (1.6) satisfies (F1) and (F2). To get (F3), it suffices to assume that $f$ grows at most linearly in the second fundamental form. More specifically,

(f3) There exists some $M>0$ such that

$$
\left|\rho f\left(-p,-\frac{R_{p}}{\rho} I\right)\right| \leqq M \quad \text { for all } \rho \in(0,1] \text { and } p \in \mathbf{R}^{n} \text { with }|p|=1 \text {. }
$$

In fact, $F_{f}$ satisfies (F3) if and only if $f$ fulfills (f3) ([29, Proposition 1.6.18]). By the definition of $F_{f},(1.6)$, it always satisfies geometricity (F5).

The growth assumption (f3) can be removed in the whole space problem by changing the notion of viscosity solutions; see e.g. [29]. Here we do not intend such generalization since we would like to keep interior interface evolution equations as simple as possible under unusual boundary condition.

Examples of interface evolution equations satisfying all of (f1)-(f3) include the mean curvature flow equation (1.7), and more generally the mean curvature flow equation with a driving force term

$$
V=H+c \quad \text { on } \Gamma_{t} \cap \Omega
$$

with a constant $c \in \mathbf{R}$. The associated $f$ for (4.20) is given by

$$
f(p, X)=\frac{1}{|p|} \operatorname{tr}\left(Q_{p}(X)\right)+c .
$$

To track an interface evolution, we assume that the initial interface $\Gamma_{0}$ and two sets $\Omega_{0}^{ \pm}$ separated by $\Gamma_{0}$ are given as follows:

(i) $\Gamma_{0} \subset \bar{\Omega}$ is a bounded closed set;

(ii) $\Omega_{0}^{ \pm} \subset \bar{\Omega}$ are disjoint, relatively open sets in $\bar{\Omega}$ such that $\Omega_{0}^{+} \cup \Omega_{0}^{-}=\bar{\Omega} \backslash \Gamma_{0}$.

Let $\Gamma_{t}, \Omega_{t}^{ \pm} \subset \bar{\Omega}$ be subsets for $t \in[0, T)$. We say that $\left\{\left(\Gamma_{t}, \Omega_{t}^{+}\right)\right\}_{t \in[0, T)}$ is a generalized solution of (1.4) and (1.10) with the initial data $\left(\Gamma_{0}, \Omega_{0}^{+}\right)$if there is a viscosity solution $u \in$ $C(\bar{\Omega} \times[0, T))$ of $(\mathrm{DB})$ such that $\Gamma_{t}=\{x \in \bar{\Omega} \mid u(x, 0)=0\}$ and $\Omega_{t}^{ \pm}=\{x \in \bar{\Omega} \mid \pm u(x, 0)>0\}$ for all $t \in[0, T)$.

Theorem 4.12. Assume (f1)-(f3). Assume (A1). Then there exists a unique generalized solution of (1.4) and (1.10) with the initial data $\left(\Gamma_{0}, \Omega_{0}^{+}\right)$.

Proof. Fix $a>0$ and define

$$
d(x)= \begin{cases}\operatorname{dist}\left(x, \Gamma_{0}\right) & \text { if } x \in \Omega_{0}^{+} \\ -\operatorname{dist}\left(x, \Gamma_{0}\right) & \text { if } x \notin \Omega_{0}^{+}\end{cases}
$$

Existence of a generalized solution is shown by solving (DB) with the initial data $u_{0}(x)=$ $\max \{\min \{d(x), a\},-a\}$. Uniqueness is a consequence of the invariance property (Theorem 4.8). Since the argument is the same as the classical one, we omit the detail; see [29, Chapter 4.1 and Chapter 4.2]. 


\section{$5 \quad$ Lipschitz continuity of solutions}

\subsection{Lipschitz estimates depending on $\beta$}

We prove that the unique solution $u$ of (DB) with a geometric $F$ is Lipschitz continuous when the initial data $u_{0}$ is regular enough.

We take an initial data $u_{0} \in C_{a}(\bar{\Omega}) \cap C^{1+1}(\bar{\Omega})$. Here we denote by $C^{1+1}(\bar{\Omega})$ the set of $u_{0} \in C^{1}(\bar{\Omega})$ whose gradient $\nabla u_{0}$ is Lipschitz continuous in $\bar{\Omega}$. Note that $u_{0}$ itself is also Lipschitz continuous in $\bar{\Omega}$. We define

$$
L_{0} \text { : the Lipschitz constant of } u_{0}, \quad L_{1} \text { : the Lipschitz constant of } \nabla u_{0} \text {. }
$$

We further set $L_{0}^{\prime}$ and $L_{0 n}$ as the Lipschitz constant of $u_{0}$ with respect to $x^{\prime}$ and $x_{n}$, respectively. More explicitly,

$$
\begin{gathered}
L_{0}^{\prime}:=\sup \left\{\frac{\left|u_{0}(x)-u_{0}(y)\right|}{|x-y|} \mid x, y \in \bar{\Omega}, x \neq y, x_{n}=y_{n}\right\}, \\
L_{0 n}:=\sup \left\{\frac{\left|u_{0}(x)-u_{0}(y)\right|}{|x-y|} \mid x, y \in \bar{\Omega}, x \neq y, x^{\prime}=y^{\prime}\right\} .
\end{gathered}
$$

We first derive some estimates for elements of semi-jets of $u_{0} \in C^{1+1}(\bar{\Omega})$. For a given $x_{0} \in \bar{\Omega}$, let us denote by $J^{2,+} u_{0}\left(x_{0}\right)\left(\right.$ resp. $\left.J^{2,-} u_{0}\left(x_{0}\right)\right)$ the set of $\left(\nabla \phi\left(x_{0}\right), \nabla^{2} \phi\left(x_{0}\right)\right) \in \mathbf{R}^{n} \times \mathbf{S}^{n}$ with $\phi \in C^{2}(\bar{\Omega})$ such that $u-\phi$ attains a local maximum (resp. local minimum) at $x_{0}$ over $\bar{\Omega}$.

Lemma 5.1. Assume that $u_{0} \in C^{1+1}(\bar{\Omega})$. Let $x \in \bar{\Omega}$ and $(p, X) \in J^{2+} u_{0}(x)$ (resp. $(p, X) \in$ $\left.J^{2,-} u_{0}(x)\right)$. Then

$$
\begin{cases}p=\nabla u_{0}(x) \text { and } X \geqq-L_{1} I\left(\text { resp. } X \leqq L_{1} I\right) & \text { if } x \in \Omega, \\ \left.p_{n} \geqq \partial_{x_{n}} u_{0}(x) \text { (resp. } p_{n} \leqq \partial_{x_{n}} u_{0}(x)\right) & \text { if } x \in \partial \Omega .\end{cases}
$$

Proof. We give the proof for $(p, X) \in J^{2,+} u_{0}(x)$. The assertions relevant to $p$, the first order derivative component, follow in an easy way since $u_{0}$ belongs to a $C^{1}$-class.

Let us fix $x \in \Omega$ and prove that $\langle X \xi, \xi\rangle \geqq-L_{1}$ for every $\xi \in \mathbf{R}^{n}$ with $|\xi|=1$. Since $(p, X) \in J^{2,+} u_{0}(x)$, we have

$$
u_{0}(x+h) \leqq u_{0}(x)+\left\langle\nabla u_{0}(x), h\right\rangle+\frac{1}{2}\langle X h, h\rangle+o\left(|h|^{2}\right) \quad \text { as } \mathbf{R}^{n} \ni h \rightarrow 0 .
$$

Note that $u_{0}(x+h)-u_{0}(x)=\int_{0}^{1}\left\langle\nabla u_{0}(x+\theta h), h\right\rangle d \theta$. Then, using this relation, CauchySchwarz inequality and the Lipschitz continuity of $\nabla u_{0}$, we observe that

$$
\begin{aligned}
\frac{1}{2}\langle X h, h\rangle+o\left(|h|^{2}\right) & \geqq \int_{0}^{1}\left\langle\nabla u_{0}(x+\theta h)-\nabla u_{0}(x), h\right\rangle d \theta \\
& \geqq-\int_{0}^{1}\left|\nabla u_{0}(x+\theta h)-\nabla u_{0}(x)\right| \cdot|h| d \theta \\
& \geqq-\int_{0}^{1} L_{1} \cdot \theta|h| \cdot|h| d \theta=-\frac{1}{2} L_{1}|h|^{2} .
\end{aligned}
$$


We now choose $h=t \xi$ for $t>0$. Then, dividing both the sides by $|h|^{2}=t^{2}$ and sending $t \rightarrow 0$, we obtain the desired inequality.

We now define

$$
\nu\left(L_{0}, L_{1}\right):=\sup \left\{\left|F\left(p, \pm L_{1} I\right)\right||0<| p \mid \leqq L_{0}\right\},
$$

which is a finite value due to the fact (4.17) and (F2). Also, define

$$
L_{\mathrm{t}}:=\max \left\{\beta L_{0 n}, \nu\left(L_{0}, L_{1}\right)\right\} \text {. }
$$

Using Lemma 5.1, we next construct a sub- and supersolution of (DB) being linear with respect to the time variable $t$.

Lemma 5.2. Assume $u_{0} \in C^{1+1}(\bar{\Omega})$. Assume $(\mathrm{F} 5)$. We define $w^{ \pm}(x, t):=u_{0}(x) \pm L_{\mathrm{t}} t$. Then $w^{-}$and $w^{+}$are, respectively, a viscosity subsolution and a viscosity supersolution of (DB).

Proof. We prove that $w^{-}$is a subsolution of (DB). It is clear that $w^{-}$satisfies the initial data. To check the other conditions, we let $(x, t) \in \bar{\Omega} \times(0, T)$ and $((p, \tau), X) \in \mathcal{P}^{2,+} w^{-}(x, t)$. By the definition of $w^{-}$, we then have $\tau=-L_{\mathrm{t}}$. Moreover, fixing the time variable, we derive $(p, X) \in J^{2,+} u_{0}(x)$.

Let $x \in \Omega$. Then Lemma 5.1 and the ellipticity of $F_{*}$ imply that

$$
\tau+F_{*}(p, X)=-L_{\mathrm{t}}+F_{*}\left(\nabla u_{0}(x), X\right) \leqq-L_{\mathrm{t}}+F_{*}\left(\nabla u_{0}(x),-L_{1} I\right) .
$$

By the definition of $L_{\mathrm{t}}$, the right-hand side is not positive. When $x \in \partial \Omega$, the boundary condition (1.2) is satisfied. In fact, noting that $p_{n} \geqq \partial_{x_{n}} u_{0}(x) \geqq-L_{0 n}$, we deduce that $\tau-\beta p_{n} \leqq-L_{\mathrm{t}}+\beta L_{0 n} \leqq 0$. The proof for $w^{+}$is similar.

In Theorem 5.3 below, we show that $L_{0}^{\prime}$ and $L_{\mathrm{t}}$ are, respectively, a Lipschitz bound for the solution $u(x, t)$ with respect to $x^{\prime}$ and $t$. Moreover the constant

$$
L_{\mathrm{n}}:=\max \left\{L_{0 n}, \frac{L_{\mathrm{t}}}{\beta}\right\}=\max \left\{L_{0 n}, \frac{\nu\left(L_{0}, L_{1}\right)}{\beta}\right\}
$$

turns to be a Lipschitz bound for $u$ with respect to $x_{n}$. Note that indexes $\mathrm{t}$ and $\mathrm{n}$ of $L$ do not mean dependence on the time-variable $t$ and the $x_{n}$-variable.

Theorem 5.3 (Lipschitz continuity). Assume $u_{0} \in C_{a}(\bar{\Omega}) \cap C^{1+1}(\bar{\Omega})$. Assume (F5). Let $u$ be the unique viscosity solution of $(\mathrm{DB})$. Then, for all $x=\left(x^{\prime}, x_{n}\right) \in \bar{\Omega}, y=\left(y^{\prime}, y_{n}\right) \in \bar{\Omega}$ and $t, s \in(0, T)$, the following estimates hold:

$$
\begin{aligned}
& |u(x, t)-u(x, s)| \leqq L_{\mathrm{t}}|t-s| \\
& \left|u\left(x^{\prime}, x_{n}, t\right)-u\left(y^{\prime}, x_{n}, t\right)\right| \leqq L_{0}^{\prime}\left|x^{\prime}-y^{\prime}\right|, \\
& \left|u\left(x^{\prime}, x_{n}, t\right)-u\left(x^{\prime}, y_{n}, t\right)\right| \leqq L_{\mathrm{n}}\left|x_{n}-y_{n}\right| .
\end{aligned}
$$


Proof. (5.1): Let $w^{ \pm}$be the functions in the statement of Lemma 5.2. Since $w^{ \pm} \in K_{a}^{ \pm}(\bar{\Omega} \times$ $[0, T)$ ), the comparison principle, Theorem 3.1 (see also Remark 3.3), implies that $w^{-} \leqq u \leqq$ $w^{+}$in $\bar{\Omega} \times(0, T)$.

For $h \in(0, T)$ let us define

$$
\tilde{w}^{ \pm}(x, t)= \begin{cases}w^{ \pm}(x, t) & \text { if } 0 \leqq t \leqq h \\ u(x, t-h) \pm L_{\mathrm{t}} h & \text { if } h \leqq t<T\end{cases}
$$

Then $\tilde{w}^{+}$is a viscosity supersolution while $\tilde{w}^{-}$is a viscosity subsolution of (DB). These assertions are obvious except at the time $t=h$. When $t=h$, they are guaranteed by the facts that $\mathcal{P}^{2, \mp} \tilde{w}^{ \pm}(x, h) \subset \mathcal{P}^{2, \mp} w^{ \pm}(x, h)$. In fact, since $\tilde{w}^{-}=w^{-}$if $t \leqq h$ and $\tilde{w}^{-} \geqq w^{-}$if $t \geqq h$, where the latter one comes from $w^{-}(x, t-h) \leqq u(x, t-h)$, we see the inclusion for $\mathcal{P}^{2,+}$. That for $\mathcal{P}^{2,-}$ follows in a similar way.

Since $\tilde{w}^{ \pm} \in K_{a}^{ \pm}(\bar{\Omega} \times[0, T))$, we therefore obtain $\tilde{w}^{-} \leqq u \leqq \tilde{w}^{+}$in $\bar{\Omega} \times(0, T)$ by comparison. At $(x, t+h)$ this gives

$$
u(x, t)-L_{\mathrm{t}} h \leqq u(x, t+h) \leqq u(x, t)+L_{\mathrm{t}} h .
$$

We have thus proved (5.1).

(5.2): For $h \in \mathbf{R}^{n-1}$ we define $v^{ \pm}(x, t):=u\left(x^{\prime}+h, x_{n}, t\right) \pm L_{0}^{\prime}|h|$. Obviously, these functions belong to $K_{a}^{ \pm}(\bar{\Omega} \times[0, T))$. Since the equation (1.1) and the boundary condition (1.2) is independent of $x^{\prime}$, the functions $v^{ \pm}$are viscosity solutions of (1.1) and (1.2). Also, at the initial time, we have $v^{-}(\cdot, 0) \leqq u_{0} \leqq v^{+}(\cdot, 0)$ in $\bar{\Omega}$. This is due to the Lipschitz continuity of $u_{0}$; that is, $u_{0}\left(x^{\prime}+h, x_{n}\right)-L_{0}^{\prime}|h| \leqq u_{0}\left(x^{\prime}, x_{n}\right) \leqq u_{0}\left(x^{\prime}+h, x_{n}\right)+L_{0}^{\prime}|h|$.

Hence the comparison principle (Theorem 3.1) implies that $v^{-} \leqq u \leqq v^{+}$in $\bar{\Omega} \times(0, T)$. By the definitions of $v^{ \pm}$, this means

$$
u\left(x^{\prime}+h, x_{n}, t\right)-L_{0}^{\prime}|h| \leqq u\left(x^{\prime}, x_{n}, t\right) \leqq u\left(x^{\prime}+h, x_{n}, t\right)+L_{0}^{\prime}|h|,
$$

which shows (5.2).

(5.3): 1. Doubling the variables. We argue by contradiction. Suppose that there are $x_{0}, y_{0} \in \bar{\Omega}$ and $t_{0} \in(0, T)$ such that $x_{0}^{\prime}=y_{0}^{\prime}$ and $M:=\left|u\left(x_{0}, t_{0}\right)-u\left(y_{0}, t_{0}\right)\right|-L_{\mathrm{n}}\left|x_{0 n}-y_{0 n}\right|>0$. Take $\delta>0$ sufficiently small so that $\left|u\left(x_{0}, t_{0}\right)-u\left(y_{0}, t_{0}\right)\right|-\tilde{L}\left|x_{0 n}-y_{0 n}\right| \geqq M / 2$ for $\tilde{L}:=L_{\mathrm{n}}+\delta$. Define a function $\Phi:(\bar{\Omega} \times[0, T])^{2} \rightarrow \mathbf{R} \cup\{-\infty\}$ by

$$
\Phi(x, t, y, s):=u(x, t)-u(y, s)-\phi(x, t, y, s)
$$

with

$$
\phi(x, t, y, s):=\tilde{L}\left|x_{n}-y_{n}\right|+\alpha\left|x^{\prime}-y^{\prime}\right|^{2}+\alpha(t-s)^{2}+\frac{\sigma}{T-t} .
$$

Here $\alpha \geqq 1, \sigma>0$ are constants such that $0<\sigma<M\left(T-t_{0}\right) / 4$, which gives

$$
\Phi\left(x_{0}, t_{0}, y_{0}, t_{0}\right) \geqq \frac{M}{2}-\frac{\sigma}{T-t_{0}}>\frac{M}{2}-\frac{M}{4}=\frac{M}{4} .
$$


Let $\hat{Z}=(\hat{x}, \hat{t}, \hat{y}, \hat{s}) \in(\bar{\Omega} \times[0, T))^{2}$ be a maximum point of $\Phi$. Since $u \in K_{a}(\bar{\Omega} \times[0, T))$, this maximum is attained in a compact set $\left(\overline{B_{R}(0)} \times[0, T]\right)^{2}$ with a suitable $R>0$. At this maximum point, we have

$$
\Phi(\hat{x}, \hat{t}, \hat{y}, \hat{s}) \geqq \Phi\left(x_{0}, t_{0}, y_{0}, t_{0}\right)>\frac{M}{4} .
$$

2. Convergence of maximum points $\hat{Z}$. Set $N:=\sup _{\bar{\Omega} \times[0, T)}|u|$. By (5.5) and the definition of $\Phi$, we then have $0<\Phi(\hat{Z}) \leqq 2 N-\alpha\left|\hat{x}^{\prime}-\hat{y}^{\prime}\right|^{2}-\alpha(\hat{t}-\hat{s})^{2}$. This gives

$$
\left|\hat{x}^{\prime}-\hat{y}^{\prime}\right|^{2} \leqq \frac{2 N}{\alpha}, \quad|\hat{t}-\hat{s}|^{2} \leqq \frac{2 N}{\alpha}
$$

Now, since $\hat{x}, \hat{y} \in \overline{B_{R}(0)}$ and $\hat{t}, \hat{s} \in[0, T)$, we may assume by (5.6) that $\left(\hat{x}^{\prime}, \hat{x}_{n}\right) \rightarrow\left(\bar{x}^{\prime}, \bar{x}_{n}\right)=$ $\bar{x} \in \bar{\Omega},\left(\hat{y}^{\prime}, \hat{y}_{n}\right) \rightarrow\left(\bar{x}^{\prime}, \bar{y}_{n}\right)=\bar{y} \in \bar{\Omega}$ and $\hat{t}, \hat{s} \rightarrow \bar{t} \in[0, T]$ as $\alpha \rightarrow \infty$.

We prove that

$$
\bar{t} \neq 0, T, \quad \bar{x}_{n} \neq \bar{y}_{n} .
$$

First, (5.5) implies that $0<2 N-\sigma /(T-\hat{t})$, and rearranging this inequality shows

$$
\hat{t}<T-\frac{\sigma}{2 N}=: T^{-} .
$$

Accordingly, $\bar{t} \leqq T^{-}<T$. Next, by (5.5) again, we see that $M / 4 \leqq u(\hat{x}, \hat{t})-u(\hat{y}, \hat{s})-\tilde{L} \mid \hat{x}_{n}-$ $\hat{y}_{n} \mid$, and sending $\alpha \rightarrow \infty$ yields

$$
\frac{M}{4} \leqq u(\bar{x}, \bar{t})-u(\bar{y}, \bar{t})-\tilde{L}\left|\bar{x}_{n}-\bar{y}_{n}\right| \cdot
$$

If $\bar{x}_{n}=\bar{y}_{n}$, the right-hand side would be 0 , and hence $\bar{x}_{n} \neq \bar{y}_{n}$. If $\bar{t}=0$, by the Lipschitz continuity of $u_{0}$ and the fact that $\tilde{L}>L_{\mathrm{n}} \geqq L_{0 n}$, we would have

$$
\frac{M}{4} \leqq u_{0}\left(\bar{x}^{\prime}, \bar{x}_{n}\right)-u_{0}\left(\bar{x}^{\prime}, \bar{y}_{n}\right)-\tilde{L}\left|\bar{x}_{n}-\bar{y}_{n}\right| \leqq L_{0 n}\left|\bar{x}_{n}-\bar{y}_{n}\right|-\tilde{L}\left|\bar{x}_{n}-\bar{y}_{n}\right| \leqq 0,
$$

a contradiction.

In what follows, we fix $\alpha \geqq 1$ sufficiently large so that

$$
\hat{t}, \hat{s} \neq 0, T, \quad \hat{x}_{n} \neq \hat{y}_{n} .
$$

In particular, the second fact implies that $\Phi$ is smooth near $\hat{Z}$.

3. Use of Crandall-Ishii lemma. We now apply the Crandall-Ishii lemma to $\Phi$ at $\hat{Z}=(\hat{x}, \hat{t}, \hat{y}, \hat{s}) \in(\bar{\Omega} \times(0, T))^{2}$. Since $\Phi$ is smooth near $\hat{Z}$, the lemma is applicable. Its conclusion guarantees that there exist $X, Y \in \mathbf{S}^{n}$ such that

$$
\begin{aligned}
\left(\left(\nabla_{x} \phi(\hat{Z}), \phi_{t}(\hat{Z})\right), X\right) & \in \overline{\mathcal{P}}^{2,+} u(\hat{x}, \hat{t}), \\
\left(\left(-\nabla_{y} \phi(\hat{Z}),-\phi_{s}(\hat{Z})\right),-Y\right) & \in \overline{\mathcal{P}}^{2,-} u(\hat{y}, \hat{s}), \\
\left(\begin{array}{cc}
X & O \\
O & Y
\end{array}\right) & \leqq A+A^{2},
\end{aligned}
$$


where $A:=\nabla_{(x, y)}^{2} \phi(\hat{Z})$. Note that $X+Y \leqq O$ for the same reason as in Step 4 in the proof of Theorem 3.1 and that

$$
\begin{aligned}
\phi_{x_{n}}(\hat{Z}) & =-\phi_{y_{n}}(\hat{Z})=\tilde{L} \frac{\hat{x}_{n}-\hat{y}_{n}}{\left|\hat{x}_{n}-\hat{y}_{n}\right|}=: p_{n} \neq 0, \\
\phi_{t}(\hat{Z}) & =2 \alpha(\hat{t}-\hat{s})+\frac{\sigma}{(T-\hat{t})^{2}}, \quad \phi_{s}(\hat{Z})=-2 \alpha(\hat{t}-\hat{s}) .
\end{aligned}
$$

Since we have already known that $u$ is Lipschitz continuous with respect to $t,(5.1)$, it follows that

$$
\left|\phi_{t}(\hat{Z})\right| \leqq L_{\mathrm{t}}, \quad\left|\phi_{s}(\hat{Z})\right| \leqq L_{\mathrm{t}} .
$$

This guarantees that the boundary condition (1.2) is violated by $\phi$. To see this, let $\hat{x}_{n}=0$. Then $p_{n}=-\tilde{L}$. Noting that $\tilde{L}=L_{\mathrm{n}}+\delta \geqq\left(L_{\mathrm{t}} / \beta\right)+\delta$, we have

$$
\phi_{t}(\hat{Z})-\beta \phi_{x_{n}}(\hat{Z}) \geqq-L_{\mathrm{t}}+\beta \tilde{L} \geqq \beta \delta>0 .
$$

Similarly, if $\hat{y}_{n}=0$,

$$
-\phi_{s}(\hat{Z})-\beta\left(-\phi_{y_{n}}(\hat{Z})\right) \leqq L_{\mathrm{t}}-\beta \tilde{L} \leqq-\beta \delta<0 .
$$

Therefore, whether $\hat{x} \in \Omega$ or $\hat{x} \in \partial \Omega$, we have by Remark 2.2

$$
\phi_{t}(\hat{Z})+F(p, X) \leqq 0, \quad-\phi_{s}(\hat{Z})+F(p,-Y) \geqq 0,
$$

where $p=\nabla_{x} \phi(\hat{Z}) \neq 0$. Subtracting these inequalities and applying the ellipticity of $F$, we see that

$$
0 \geqq \phi_{t}(\hat{Z})+\phi_{s}(\hat{Z})+F(p, X)-F(p,-Y) \geqq \frac{\sigma}{(T-\hat{t})^{2}}+0>0 .
$$

This is a contradiction.

Remark 5.4. In view of the proof, we notice that it suffices to assume that $u_{0} \in C_{a}(\bar{\Omega})$ satisfies $L_{0}^{\prime}<\infty$ in order to prove (5.2), while $C^{1+1}$-regularity of $u_{0}$ is used to derive (5.1). Also, (5.3) is proved by making use of (5.1).

\subsection{Lipschitz estimates independent of $\beta$}

The Lipschitz bound with respect to $x_{n}$ given in (5.3) of Theorem 5.3 depends on $\beta$, and this is derived for smooth initial data $u_{0}$. We next prove that, for rather restrictive initial data and equations, but for less regular initial data, the Lipschitz bound is uniform in $\beta$. This enables us to obtain the unique Lipschitz continuous viscosity solution of (DB) with $\beta=0$; see Theorem 6.6.

We will impose the following assumptions. They will be used to construct barrier functions independent of $\beta$; see Step 1 in the proof of Theorem 5.7. First, the initial data $u_{0} \in C_{a}(\bar{\Omega})$ is supposed to be independent of $x^{\prime}$ on $\partial \Omega$ and Lipschitz continuous with respect to $x_{n}$ from the boundary. Namely,

(i) $u_{0}\left(x^{\prime}, 0\right) \equiv a$ for all $x^{\prime} \in \mathbf{R}^{n-1}$; 
(ii) there exists some $L_{0 n}^{*}>0$ such that

$$
\left|u_{0}\left(x^{\prime}, x_{n}\right)-u_{0}\left(x^{\prime}, 0\right)\right| \leqq L_{0 n}^{*} x_{n} \quad \text { for all } x \in \bar{\Omega} .
$$

We next assume that $F=F(p, X)$ satisfies (F6) below. In what follows, we represent a vector $p \in \mathbf{R}^{n}$ and a matrix $X \in \mathbf{S}^{n}$ as

$$
p=\left(\begin{array}{c}
p^{\prime} \\
p_{n}
\end{array}\right), \quad X=\left(\begin{array}{cc}
X^{\prime} & \vec{x} \\
\vec{x}^{t} & x_{n n}
\end{array}\right)
$$

with $p^{\prime} \in \mathbf{R}^{n-1}, p_{n} \in \mathbf{R}, X^{\prime} \in \mathbf{S}^{n-1}, \vec{x} \in \mathbf{R}^{n-1}$ and $x_{n n} \in \mathbf{R}$.

(F6) $F(p, O)=0$ for all $p \in \mathbf{R}^{n} \backslash\{0\}$ such that $p^{\prime}=0$.

For example, it is easily seen that the mean curvature flow operator (1.9) satisfies (F6). Remark 5.5. If $F$ satisfies (F5) and (F6), then

$$
\begin{array}{ll}
F(p, X)=0 & \text { for all }(p, X) \in\left(\mathbf{R}^{n} \backslash\{0\}\right) \times \mathbf{S}^{n} \\
& \text { such that } p^{\prime}=0, X^{\prime}=O \text { and } \vec{x}=0 .
\end{array}
$$

To see this, let us fix such $(p, X)$. By (F5) we have $\lambda F(p, X)=F(\lambda p, \lambda X+\sigma p \otimes p)$ for any $\lambda>0$ and $\sigma \in \mathbf{R}$. We now choose $\sigma=-\lambda x_{n n} / p_{n}^{2}$, so that $\lambda X+\sigma p \otimes p=O$. Then, by (F6), we see that $\lambda F(p, X)=F(\lambda p, O)=0$. This shows that $F(p, X)=0$.

Remark 5.6. Another possible condition on $F$ which is more general than (F6) is

(F6)' There is a constant $\mu \in \mathbf{R}$ such that $F(p, O)=\mu$ for all $p \in \mathbf{R}^{n} \backslash\{0\}$ such that $p^{\prime}=0$.

However, it turns out that $\mu$ should be 0 under (F5). In fact, $(\mathrm{F} 6)^{\prime}$ gives $F_{*}(0, O) \leqq \mu \leqq$ $F^{*}(0, O)$. Since $(4.16)$ holds under (F5), we conclude that $\mu=0$.

Theorem 5.7 (Lipschitz continuity). Assume $u_{0} \in C_{a}(\bar{\Omega})$ and (A2). Assume (F5) and (F6). Let $u$ be the unique viscosity solution of $(\mathrm{DB})$. Then, for all $x=\left(x^{\prime}, x_{n}\right) \in \bar{\Omega}, y=\left(x^{\prime}, y_{n}\right) \in \bar{\Omega}$ and $t \in(0, T)$, the following estimate holds:

$$
\left|u\left(x^{\prime}, x_{n}, t\right)-u\left(x^{\prime}, y_{n}, t\right)\right| \leqq L_{0 n}^{*}\left|x_{n}-y_{n}\right| .
$$

Proof. 1. Barriers (Lipschitz continuity from the boundary). We prove that

$$
\left|u\left(x^{\prime}, x_{n}, t\right)-u\left(x^{\prime}, 0, t\right)\right| \leqq L_{0 n}^{*} x_{n} \quad \text { for all }(x, t) \in \bar{\Omega} \times(0, T) .
$$

Set $w^{ \pm}(x, t)=a \pm L_{0 n}^{*} g\left(x_{n}\right)$ with $g\left(x_{n}\right)=\sqrt{x_{n}^{2}+\varepsilon}$ for $\varepsilon>0$. Clearly, $w^{ \pm} \in K_{a}^{ \pm}(\bar{\Omega} \times[0, T))$. We show that $w^{ \pm}$are classical solutions of (1.1) and (1.2). In the following, we only study $w^{-}$since the same argument applies to $w^{+}$.

Let $t>0$. If $x \in \Omega$, we see by (5.10) that

$$
\begin{aligned}
& w_{t}^{-}(x, t)+F_{*}\left(\nabla w^{-}(x, t), \nabla^{2} w^{-}(x, t)\right) \\
& =0+F\left(\left(\begin{array}{c}
0 \\
-L_{0} g^{\prime}\left(x_{n}\right)
\end{array}\right),\left(\begin{array}{cc}
O & 0 \\
0 & -L_{0} g^{\prime \prime}\left(x_{n}\right)
\end{array}\right)\right)=0 .
\end{aligned}
$$


If $x \in \partial \Omega$, then

$$
w_{t}^{-}(x, t)-\beta w_{x_{n}}^{-}(x, t)=0-\beta\left(-L_{0} g^{\prime}(0)\right)=0 .
$$

Thus $w^{-}$is a classical solution.

When $t=0$, the fact $g\left(x_{n}\right) \geqq x_{n}$ and the assumption (A2) on $u_{0}$ give

$$
w^{-}(x, 0) \leqq a-L_{0 n}^{*} x_{n}=u_{0}\left(x^{\prime}, 0\right)-L_{0 n}^{*} x_{n} \leqq u_{0}\left(x^{\prime}, x_{n}\right) .
$$

In the same manner, we obtain $w^{+}(x, 0) \geqq u_{0}\left(x^{\prime}, x_{n}\right)$. Therefore, the comparison principle (Theorem 3.1) implies that $w^{-} \leqq u \leqq w^{+}$. Sending $\varepsilon \rightarrow 0$, we obtain

$$
a-L_{0 n}^{*} x_{n} \leqq u(x, t) \leqq a+L_{0 n}^{*} x_{n} \quad \text { for all }(x, t) \in \bar{\Omega} \times(0, T) .
$$

Putting $x_{n}=0$ in the above, we find $u\left(x^{\prime}, 0, t\right)=a$. Finally we plug this into the above inequalities to conclude (5.12).

2. Proof of (5.11). We carry out the same argument as in the proof of (5.3) in Theorem 5.3. Here the only difference is that we replace $\tilde{L}$ by $L_{0 n}^{*}$ in the definition of $\phi,(5.4)$. Then the same discussions as in Step 1 and 2 work without any changes, and we have the limit $\bar{x}=\left(\bar{x}^{\prime}, \bar{x}_{n}\right) \in \bar{\Omega}$ and $\bar{y}=\left(\bar{x}^{\prime}, \bar{y}_{n}\right) \in \bar{\Omega}$. Now we further prove that

$$
\bar{x}_{n}, \bar{y}_{n} \neq 0 .
$$

Suppose $\bar{y}_{n}=0$. Then, applying (5.12) to (5.7) with $L_{0 n}^{*}$ instead of $\tilde{L}$, we would have

$$
\frac{M}{4} \leqq u\left(\bar{x}^{\prime}, \bar{x}_{n}, \bar{t}\right)-u\left(\bar{x}^{\prime}, 0, \bar{t}\right)-L_{0 n}^{*} \bar{x}_{n} \leqq 0 .
$$

This is a contradiction, and so $\bar{y}_{n} \neq 0$. Similarly, we obtain $\bar{x}_{n} \neq 0$. Hence we may assume that

$$
\hat{x}_{n}, \hat{y}_{n} \neq 0
$$

in addition to (5.8).

We apply Crandall-Ishii lemma at $\hat{Z}=(\hat{x}, \hat{t}, \hat{y}, \hat{s})$. Since $\hat{x}, \hat{y} \notin \partial \Omega$ by (5.13), the resulting viscosity inequalities are those for the equation (1.1). Namely, we have (5.9) with $p=$ $\nabla_{x} \phi(\hat{Z}) \neq 0$ and $X, Y \in \mathbf{S}^{n}$ such that $X+Y \leqq O$. As before, we arrive at a contradiction.

\section{Asymptotic behavior of solutions with respect to $\beta$}

\subsection{Half-relaxed limits}

We study the asymptotic behavior of solutions $u^{\beta}$ to (DB) as $\beta \rightarrow 0$ or $\beta \rightarrow \infty$. For this purpose, we study the upper half-relaxed limits $\bar{u}$ and the lower half-relaxed limits $\underline{u}$ of the solutions $u^{\beta}$ to (DB), which are defined as, in the case where $\beta \rightarrow 0$,

$$
\begin{aligned}
\bar{u}(x, t) & =\limsup _{\beta \rightarrow 0} u^{\beta}(x, t) \\
& :=\lim _{\delta \rightarrow 0} \sup \left\{u^{\beta}(y, s)|| x-y|<\delta,| t-s \mid<\delta, 0<\beta<\delta\right\}, \\
\underline{u}(x, t) & =\liminf _{\beta \rightarrow 0} u^{\beta}(x, t) \\
& :=\lim _{\delta \rightarrow 0} \inf \left\{u^{\beta}(y, s)|| x-y|<\delta,| t-s \mid<\delta, 0<\beta<\delta\right\} .
\end{aligned}
$$


When we send $\beta \rightarrow \infty$, we replace " $0<\beta<\delta$ " by " $1 / \delta<\beta$ ".

By stability results for viscosity solutions ([10, Lemma 6.1, Remarks 6.2 and 6.3]), it is known that $\bar{u}$ and $\underline{u}$ are, respectively, a viscosity subsolution and a viscosity supersolution of the corresponding limit problem with the boundary condition $u_{t}(x, t)=0$ on $\partial \Omega \times(0, T)$ as $\beta \rightarrow 0$ and $-u_{x_{n}}(x, t)=0$ on $\partial \Omega \times(0, T)$ as $\beta \rightarrow \infty$, provided that $-\infty<\bar{u}<\infty$ and $-\infty<\underline{u}<\infty$. If we further know that $\bar{u}=\underline{u}=: u$, then we conclude that $u^{\beta}$ converges to $u$ locally uniformly; see [10, Remark 6.4]. Usually, the fact that $\bar{u}=\underline{u}$ is guaranteed by a comparison principle to the limit problem.

For (DB), the fact that $-\infty<\underline{u} \leqq \bar{u}<\infty$ follows from (4.15) since the left- and right-hand sides of (4.15) do not depend on $\beta$. We thus have

Proposition 6.1. Assume $u_{0} \in C_{a}(\bar{\Omega})$. Assume (F5). Then, for both the case $\beta \rightarrow 0$ and $\beta \rightarrow \infty$, we have $-\infty<\underline{u} \leqq \bar{u}<\infty$ in $\bar{\Omega} \times[0, T)$ and $\bar{u}(\cdot, 0)=\underline{u}(\cdot, 0)=u_{0}$ in $\bar{\Omega}$. Moreover, $\bar{u}$ and $\underline{u}$ are continuous on $\bar{\Omega} \times\{0\}$.

\subsection{The limit as $\beta \rightarrow \infty$}

In this case, the limit problem is

$$
(\mathrm{NE}) \begin{cases}u_{t}(x, t)+F\left(\nabla u(x, t), \nabla^{2} u(x, t)\right)=0 & \text { in } \Omega \times(0, T), \\ -u_{x_{n}}(x, t)=0 & \text { on } \partial \Omega \times(0, T), \\ u(x, 0)=u_{0}(x) & \text { in } \bar{\Omega} .\end{cases}
$$

The comparison principle for this Neumann boundary value problem (NE) is a classical result ([45, Theorem 2.1]). We therefore obtain

Theorem 6.2 (Convergence). Assume $u_{0} \in C_{a}(\bar{\Omega})$. Assume (F5). Let $u^{\beta}$ and $u$ be the unique viscosity solutions of $(\mathrm{DB})$ and $(\mathrm{NE})$ respectively. Then $u^{\beta}$ converges to $u$ locally uniformly in $\bar{\Omega} \times[0, T)$ as $\beta \rightarrow \infty$.

\subsection{The limit as $\beta \rightarrow 0$ (I)}

Unfortunately, viscosity solutions to the limit problem

$$
(\mathrm{DB})_{0} \begin{cases}u_{t}(x, t)+F\left(\nabla u(x, t), \nabla^{2} u(x, t)\right)=0 & \text { in } \Omega \times(0, T), \\ u_{t}(x, t)=0 & \text { on } \partial \Omega \times(0, T), \\ u(x, 0)=u_{0}(x) & \text { in } \bar{\Omega}\end{cases}
$$

are not unique as is shown in [2, Example A.5]. Moreover, in the viscosity sense, the dynamic boundary condition (6.1) may be different from a Dirichlet condition

$$
u(x, t)=u_{0}(x) \quad \text { on } \partial \Omega \times(0, T)
$$

obtained by the integration of (6.1); see [12, Section 5] and [30, Section 5.3].

For this reason, we restrict ourselves to the case where the limits of $u^{\beta}$ satisfy (6.2) in the strong (classical) sense so that the comparison principle is applicable. Here we say that $u$ satisfies $(6.2)$ in the strong sense if $u(x, t)=u_{0}(x)$ for all $(x, t) \in \partial \Omega \times(0, T)$.

We will give a sufficient condition on the initial data which guarantees the boundary condition in the strong sense. We assume existence of barrier functions as follows: 
(A3) For all $x_{0} \in \partial \Omega$ and $\eta>0$, there exist functions $\phi^{ \pm} \in C(\bar{\Omega})$ such that

(i) $\phi^{-} \leqq u_{0} \leqq \phi^{+}$in $\bar{\Omega}$;

(ii) $u_{0}\left(x_{0}\right)-\eta \leqq \phi^{-}\left(x_{0}\right)$ and $\phi^{+}\left(x_{0}\right) \leqq u_{0}\left(x_{0}\right)+\eta$;

(iii) $\phi^{+}$and $\phi^{-}$are, respectively, a viscosity supersolution and a viscosity subsolution of

$$
F\left(\nabla \phi, \nabla^{2} \phi\right)=0 \quad \text { in } \Omega ;
$$

(iv) there exist some $L^{ \pm}>0$ such that

$$
\left|\phi^{ \pm}\left(x^{\prime}, x_{n}\right)-\phi^{ \pm}\left(x^{\prime}, 0\right)\right| \leqq L^{ \pm} x_{n} \quad \text { for all } x \in \bar{\Omega} .
$$

Under (A3) it turns out that $u^{\beta}$ converges to the unique solution of

$$
\text { (DI) } \begin{cases}u_{t}(x, t)+F\left(\nabla u(x, t), \nabla^{2} u(x, t)\right)=0 & \text { in } \Omega \times(0, T), \\ u(x, t)=u_{0}(x) \quad \text { in the strong sense) } & \text { on } \partial \Omega \times(0, T), \\ u(x, 0)=u_{0}(x) & \text { in } \bar{\Omega} .\end{cases}
$$

By the classical theory (e.g. [10, Theorem 8.2]), the comparison principle is true for a viscosity sub- and supersolution of (DI).

Proposition 6.3. Assume $u_{0} \in C_{a}(\bar{\Omega})$ and (A3). Assume (F5). Let $u^{\beta}$ be the unique viscosity solutions of (DB). Then $\bar{u}$ and $\underline{u}$ satisfy (6.3).

Proof. We prove that $\bar{u}$ satisfies (6.3). The proof for $\underline{u}$ is omitted since it is parallel. Fix $\left(x_{0}, t_{0}\right) \in \partial \Omega \times(0, T)$. We also fix $\eta>0$ and take the functions $\phi^{ \pm}$in (A3). We further fix $\varepsilon>0$ and define $v^{ \pm}(x, t):= \pm \varepsilon t+\phi^{ \pm}(x)$. Then $v^{+}$and $v^{-}$are, respectively, a viscosity supersolution and a viscosity subsolution of (DB) provided that $0<\beta<\varepsilon / L^{ \pm}$, where $L^{ \pm}$ are the constants in (A3)-(iv). Indeed, the equation (1.1) is easy to check by (A3)-(iii). If $((p, \tau), X) \in \mathcal{P}^{2,-} v^{+}(x, t)$ for $x \in \partial \Omega$, then we have $\tau=\varepsilon$ and $\left|p_{n}\right| \leqq L^{+}$. Therefore

$$
\tau-\beta p_{n} \geqq \varepsilon-\beta L^{+}>0
$$

by the choice of $\beta$. Accordingly, the boundary condition (1.2) is satisfied. The proof for $v^{-}$ is similar.

Since $v^{-}(\cdot, 0) \leqq u_{0} \leqq v^{+}(\cdot, 0)$ in $\bar{\Omega}$ by (A3)-(i), the comparison principle implies that $v^{-} \leqq u^{\beta} \leqq v^{+}$in $\bar{\Omega} \times(0, T)$. Taking lim $\sup _{\beta \rightarrow 0}^{*}$ in these inequalities and then sending $\varepsilon \rightarrow 0$, we obtain $\phi^{-} \leqq \bar{u} \leqq \phi^{+}$in $\bar{\Omega} \times(0, T)$. In particular, using (A3)-(ii), we have

$$
u_{0}\left(x_{0}\right)-\eta \leqq \phi^{-}\left(x_{0}\right) \leqq \bar{u}\left(x_{0}, t_{0}\right) \leqq \phi^{+}\left(x_{0}\right) \leqq u_{0}\left(x_{0}\right)+\eta .
$$

Since $\eta>0$ is arbitrary, this shows that $\bar{u}\left(x_{0}, t_{0}\right)=u_{0}\left(x_{0}\right)$. The proof is complete.

As a consequence of Proposition 6.3, we obtain

Theorem 6.4 (Convergence). Assume $u_{0} \in C_{a}(\bar{\Omega})$ and (A3). Assume (F5). Let $u^{\beta}$ and $u$ be the unique viscosity solutions of (DB) and (DI) respectively. Then $u^{\beta}$ converges to $u$ locally uniformly in $\bar{\Omega} \times[0, T)$ as $\beta \rightarrow 0$.

Proof. Since $\bar{u}$ and $\underline{u}$ are, respectively, a viscosity subsolution and a viscosity supersolution of (1.1) with (1.3) and since they satisfy (6.3) by Proposition 6.3, the comparison principle for (DI) yields $\bar{u} \leqq \underline{u}$ in $\bar{\Omega} \times[0, T)$. Therefore $\bar{u}=\underline{u}$ in $\bar{\Omega} \times[0, T)$, which implies the locally uniform convergence as required. 


\subsection{The limit as $\beta \rightarrow 0$ (II)}

We provide another convergence result as $\beta \rightarrow 0$. Although a comparison principle for $(\mathrm{DB})_{0}$ is not true in general, as is pointed out in [2, Lemma 3.2], a Lipschitz continuous suband supersolution are comparable. For our problem $(\mathrm{DB})_{0}$, it suffices to assume Lipschitz continuity with respect to $x_{n}$ from the boundary; namely (5.12).

Theorem 6.5 (Comparison principle). Let $u: \bar{\Omega} \times[0, T) \rightarrow \mathbf{R}$ be a viscosity subsolution of $(\mathrm{DB})_{0}$ which is assumed to be bounded from above. Let $v: \bar{\Omega} \times[0, T) \rightarrow \mathbf{R}$ be a viscosity supersolution of $(\mathrm{DB})_{0}$ which is assumed to be bounded from below. Assume (3.1) and that both $u$ and $v$ satisfy (5.12) with some $L>0$ instead of $L_{0 n}^{*}$. If $u^{*}(\cdot, 0) \leqq v_{*}(\cdot, 0)$ in $\bar{\Omega}$, then $u^{*} \leqq v_{*}$ in $\bar{\Omega} \times(0, T)$.

Proof. For $\varepsilon>0$ we define $u_{\varepsilon}(x, t):=u(x, t)-\varepsilon L t$ and $v_{\varepsilon}(x, t):=v(x, t)+\varepsilon L t$. Then it is easily seen that $u_{\varepsilon}$ and $v_{\varepsilon}$ are, respectively, a viscosity subsolution and a viscosity supersolution of (DB) with $\beta=\varepsilon$. Moreover, $u_{\varepsilon}$ and $v_{\varepsilon}$ satisfy (3.1). Since $\left(u_{\varepsilon}\right)^{*}(x, 0)=$ $u^{*}(x, 0) \leqq v_{*}(x, 0)=\left(v_{\varepsilon}\right)_{*}(x, 0)$, Theorem 3.1 implies that $\left(u_{\varepsilon}\right)^{*} \leqq\left(v_{\varepsilon}\right)_{*}$. That is, $u^{*}-\varepsilon L t \leqq$ $v_{*}+\varepsilon L t$. Finally, sending $\varepsilon \rightarrow 0$ gives the desired conclusion.

Under the assumptions of Theorem 5.7, the solutions $u^{\beta}$ of (DB) satisfy (5.12) with $L_{0 n}^{*}$ being uniform in $\beta>0$. We therefore have the following convergence result:

Theorem 6.6 (Convergence). Assume $u_{0} \in C_{a}(\bar{\Omega})$ and (A2). Assume (F5) and (F6). Let $u^{\beta}$ be the unique viscosity solution of $(\mathrm{DB})$. Then $u^{\beta}$ converges to $u$ locally uniformly in $\bar{\Omega} \times[0, T)$ as $\beta \rightarrow 0$, where $u$ is the unique viscosity solution of $(\mathrm{DB})_{0}$ satisfying $(5.12)$.

Proof. Since $u^{\beta}$ satisfies (5.12) with $L_{0 n}^{*}$ independent of $\beta>0$, the upper and lower halfrelaxed limits $\bar{u}$ and $\underline{u}$ also satisfy $(5.12)$. Theorem 6.5 is thus applicable to them, and so $\bar{u} \leqq \underline{u}$ in $\bar{\Omega} \times[0, T)$. This gives the result.

\section{Acknowledgment}

The authors are grateful to Professor Moto-Hiko Sato who initiated this project with useful suggestions.

The work of the first author was partly supported by Japan Society for the Promotion of Science (JSPS) through the grants No. 26220702 (Kiban S), No. 17H01091 (Kiban A) and No. $16 \mathrm{H} 03948$ (Kiban B). The work of the second author was partly supported by JSPS through the grant No. 16K17621 (Wakate B) and by The Sumitomo Foundation through Grant for Basic Science Research Projects No. 150973 and Inamori Foundation through Research Grants.

\section{References}

[1] H. Abels, H. Garcke, L. Müller, Local well-posedness for volume-preserving mean curvature and Willmore flows with linear tension, Math. Nachr. 289 (2016), 136-174. 
[2] E. S. Al-Aidarous, E. O. Alzahrani, H. Ishii, A. M. M. Younas, Asymptotic analysis for the eikonal equation with the dynamical boundary conditions, Math. Nachr. 287 (2014), 1563-1588.

[3] H. Amann, M. Fila, A Fujita-type theorem for the Laplace equation with a dynamical boundary condition, Acta Math. Univ. Comenian. 66 (1997), 321-328.

[4] S. Angenent, M. E. Gurtin, General contact-angle conditions with and without kinetics, Quart. Appl. Math. 54 (1996), 557-569.

[5] G. Barles, Fully nonlinear Neumann type boundary conditions for second-order elliptic and parabolic equations, J. Differential Equations 106 (1993), 90-106.

[6] G. Barles, Nonlinear Neumann boundary conditions for quasilinear degenerate elliptic equations and applications, J. Differential Equations 154 (1999), 191-224.

[7] G. Barles, H. Ishii, H. Mitake, On the large time behavior of solutions of HamiltonJacobi equations associated with nonlinear boundary conditions, Arch. Ration. Mech. Anal. 204 (2012), 515-558.

[8] Y.-G. Chen, Y. Giga, S. Goto, Uniqueness and existence of viscosity solutions of generalized mean curvature flow equations, J. Differential Geom. 33 (1991), 749-786.

[9] P. Colli, T. Fukao, The Allen-Cahn equation with dynamic boundary conditions and mass constraints, Math. Methods Appl. Sci. 38 (2015), 3950-3967.

[10] M. G. Crandall, H. Ishii, P.-L. Lions, User's guide to viscosity solutions of second order partial differential equations, Bull. Amer. Math. Soc. (N.S.) 27 (1992), 1-67.

[11] R. Denk, J. Prüss, R. Zacher, Maximal $L_{p}$-regularity of parabolic problems with boundary dynamics of relaxation type, J. Funct. Anal. 255 (2008), 3149-3187.

[12] C. M. Elliott, Y. Giga, S. Goto, Dynamic boundary conditions for Hamilton-Jacobi equations, SIAM J. Math. Anal. 34 (2003), 861-881.

[13] J. Escher, Nonlinear elliptic systems with dynamic boundary conditions, Math. Z. 210 (1992), 413-439.

[14] J. Escher, Quasilinear parabolic systems with dynamical boundary conditions, Comm. Partial Differential Equations 18 (1993), 1309-1364.

[15] J. Escher, Smooth solutions of nonlinear elliptic systems with dynamic boundary conditions, Lect. Notes PureAppl. Math. 155 (1994), 173-183.

[16] L. C. Evans, H. M. Soner, P. E. Souganidis, Phase transitions and generalized motion by mean curvature, Comm. Pure Appl. Math. 45 (1992), 1097-1123.

[17] L. C. Evans, J. Spruck, Motion of level sets by mean curvature. I, J. Differential Geom. 33 (1991), 635-681. 
[18] M. Fila, K. Ishige, T. Kawakami, Convergence to the Poisson kernel for the Laplace equation with a nonlinear dynamical boundary condition, Commun. Pure Appl. Anal. 11 (2012), 1285-1301.

[19] M. Fila, K. Ishige, T. Kawakami, Large-time behavior of small solutions of a twodimensional semilinear elliptic equation with a dynamical boundary condition, Asymptot. Anal. 85 (2013), 107-123.

[20] M. Fila, K. Ishige, T. Kawakami, Large-time behavior of solutions of a semilinear elliptic equation with a dynamical boundary condition, Adv. Differential Equations 18 (2013), 69-100.

[21] M. Fila, K. Ishige, T. Kawakami, Existence of positive solutions of a semilinear elliptic equation with a dynamical boundary condition, Calc. Var. Partial Differential Equations 54 (2015), 2059-2078.

[22] M. Fila, K. Ishige, T. Kawakami, Minimal solutions of a semilinear elliptic equation with a dynamical boundary condition, J. Math. Pures Appl. 105 (2016), 788-809.

[23] M. Fila, K. Ishige, T. Kawakami, An exterior nonlinear elliptic problem with a dynamical boundary condition, Rev. Mat. Complut. 30 (2017), 281-312.

[24] M. Fila, K. Ishige, T. Kawakami, The large diffusion limit for the heat equation with a dynamical boundary condition, in preparation.

[25] M. Fila, P. Poláčik, Global nonexistence without blow-up for an evolution problem Math. Z. 232 (1999), 531-545.

[26] C. G. Gal, M. Grasselli, The non-isothermal Allen-Cahn equation with dynamic boundary conditions, Discrete Contin. Dyn. Syst. 22 (2008), 1009-1040.

[27] M.-H. Giga, Y. Giga, Evolving graphs by singular weighted curvature, Arch. Ration. Mech. Anal. 141 (1998), 117-198.

[28] M.-H. Giga, Y. Giga, N. Požár, Periodic total variation flow of non-divergence type in $\mathbb{R}^{n}$, J. Math. Pures Appl. (9) 102 (2014), 203-233.

[29] Y. Giga, Surface evolution equations: A level set approach, Monographs in Mathematics, 99, Birkhauser Verlag, Basel, 2006, xii+264 pp.

[30] Y. Giga, N. Hamamuki, Hamilton-Jacobi equations with discontinuous source terms, Comm. Partial Differential Equations 38 (2013), 199-243.

[31] Y. Giga, F. Onoue, K. Takasao, A varifold formulation of mean curvature flow with Dirichlet or dynamic boundary condition, in preparation.

[32] Y. Giga, N. Požár, Approximation of general facets by regular facets with respect to anisotropic total variation energies and its application to the crystalline mean curvature flow, Comm. Pure Appl. Math., published online (2018). 
[33] S. Goto, Generalized motion of hypersurfaces whose growth speed depends superlinearly on the curvature tensor, Differential Integral Equations 7 (1994), 323-343.

[34] N. Hamamuki, Q. Liu, A deterministic game interpretation for level-set curvature flow equations with dynamic boundary conditions, in preparation.

[35] N. Hamamuki, Q. Liu, A game-theoretic approach to dynamic boundary problems for fully nonlinear parabolic equations and applications, in preparation.

[36] T. Ilmanen, P. Sternberg, W. P. Ziemer, Equilibrium solutions to generalized motion by mean curvature, J. Geom. Anal. 8 (1998), 845-858.

[37] H. Ishii, Perron's method for Hamilton-Jacobi equations, Duke Math. J. 55 (1987), 369-384.

[38] H. Ishii, M.-H. Sato, Nonlinear oblique derivative problems for singular degenerate parabolic equations on a general domain, Nonlinear Anal. 57 (2004), 1077-1098.

[39] M. Katsoulakis, G. T. Kossioris, F. Reitich, Generalized motion by mean curvature with Neumann conditions and the Allen-Cahn model for phase transitions, J. Geom. Anal. 5 (1995), 255-279.

[40] M. Kirane, E. Nabana, S. I. Pokhozhaev, On the absence of solutions for elliptic systems with dynamic boundary conditions, Differ. Equ. 38 (2002), 808-815.

[41] M. Kirane, E. Nabana, S. I. Pokhozhaev, Nonexistence of global solutions to an elliptic equation with a dynamical boundary condition, Bol. Soc. Parana. Mat. (3) 22 (2004), $9-16$.

[42] S. Osher, J. A. Sethian, Fronts propagating with curvature-dependent speed: algorithms based on Hamilton-Jacobi formulations, J. Comput. Phys. 79 (1988), 12-49.

[43] T. Otha, D. Jasnow, K. Kawasaki, Universal scaling in the motion of random interface, Physics Review Letters 49 (1982), 1223-1226.

[44] M.-H. Sato, Interface evolution with Neumann boundary condition, Adv. Math. Sci. Appl. 4 (1994), 249-264.

[45] M.-H. Sato, Capillary problem for singular degenerate parabolic equations on a half space, Differential Integral Equations 9 (1996), 1213-1224.

[46] J. Sprekels, H. Wu, A note on parabolic equation with nonlinear dynamical boundary condition Nonlinear Anal. 72 (2010), 3028-3048.

[47] P. Sternberg, W. P. Ziemer, Generalized motion by curvature with a Dirichlet condition, J. Differential Equations 114 (1994), 580-600.

[48] J. L. Vázquez, E. Vitillaro, Heat equation with dynamical boundary conditions of reactive type, Comm. Partial Differential Equations 33 (2008), 561-612. 